\title{
Theory of Matching Rules for the 3-Dimensional Penrose Tilings
}

\author{
A. Katz \\ Institute for Theoretical Physics, UCSB, Santa Barbara, CA 93106, USA \\ Centre de Physique Théorique, Ecole Polytechnique, F-91128 Palaiseau Cedex, France
}

\begin{abstract}
We consider packings of the two Ammann rhombohedra used for tiling the three dimensional space. We define decorations for the facets of the rhombohedra. Using elementary algebraic topology, we prove that any tiling by these rhombohedra with matching decorations is a quasiperiodic Penrose tiling. The proof does not involve any reference to self similarity.
\end{abstract}

\section{Introduction}

Since their invention by Roger Penrose, his well-known aperiodic and five-fold symmetric tilings of the planes have motivated numerous works [1-3]. See [4] for a review on the Penrose and related tilings. The construction and therefore a possible description of these tilings is controlled by their strong self-similarity properties, known as "inflation" and "deflation", which give access to some of their main properties, and specifically to their aperiodicity. On the other hand, these tilings can be obtained through a local "growth process" constrained by the socalled matching rules: there exists a set of decorations of the edges of the tiles (which can of course be realized in many different ways), such that any infinite tilings in which the decorations of the edges of any adjacent tiles coincide is a Penrose tiling.

Although the Penrose tilings had early attracted the interest of some crystallographers [5] speculating on their possible implications for solid state physics (and who have first empirically observed their quasiperiodicity using optical transforms), it is the discovery of icosahedral quasicrystals [6] in rapidly cooled alloys of Aluminium and Manganese which has triggered an intense interest for these structures in the solid state physics community. See [7] for a general review. Three dimensional analogues of the Penrose tilings were soon devised [8,9] and their quasiperiodicity proved [9]. These 3-dimensional tilings are packings of two rhombohedra with identical facets known as Ammann rhombohedra, and they are icosahedrally symmetric in the same loose sense as the Penrose tilings are five-fold symmetric.

Up to now, only global methods such as the strip projection method are known to construct these tilings, and it was interesting to look for matching rules 
analogous to those relative to the 2-dimensional case. In fact, our interest for this question relies mainly on physical grounds: the quasicrystals are known to grow fast from the melt, in such a way that it seems unlikely that their (meta)stability could depend only on "large scale" features of their structure (with respect to the atomic scale). On the contrary, it seems likely that the growth of quasicrystals is mainly governed by local phenomena. In this respect, the inexistence of matching rules of reasonable short range for the 3-dimensional Penrose tilings would result in an hardly understandable situation. Of course, we do not mean that atoms obey matching rules, nor even that there exists a specific relation between the atomic structure of the quasicrystals and the 3-dimensional Penrose tilings, but only that the existence of matching rules supports the idea of a local growth process resulting in a global quasiperiodic ordering.

Such matching rules do exist, as proved in this paper. They consist in the matching of decorations defined on the facets of the two rhombohedra, in complete analogy with the 2-dimensional case. However, we need fourteen different decorations for the thick rhombohedron, and eight for the flat one, in sharp contrast with the only two Penrose rhombs (it is not proved that this number cannot be reduced).

The paper is organized as follows:

In the next section, we recall the strip projection method used to construct quasiperiodic tilings of the 3-dimensional space by means of the two Ammann rhombohedra, which is the natural setting to define our decorations. This is done in the third section, where we first define the decoration of the facets and then study the decorated rhombohedra which result from this decoration. In order to introduce homological methods, we sketch in the fourth section another description of the tilings, known as the cut method, in which the quasiperiodic tilings are obtained through a plane section of a periodic "oblique" tiling in six dimensions. Then we state in the fifth section some elementary results of algebraic topology, and show their relevance for our problem. The sixth section is devoted to a more precise study of the geometry of the oblique tiling, which prepares for the proof of our main theorem, given in the seventh section. The last section is devoted to some comments concerning the limitations of the theory and its applicability to other cases.

\section{The Strip Projection}

2.1. Let us first briefly recall the strip projection method used to construct 3-dimensional Penrose tilings. The reader is referred to [9] for a full exposition on the subject. They can be obtained through specializations of a general method which yields quasiperiodic tilings in any dimension. We describe it only in the icosahedral case. Let $\mathbb{Z}^{6}$ be the canonical hypercubic lattice in $\mathbb{R}^{6}$ with basis $\left\{\varepsilon_{1}, \ldots, \varepsilon_{6}\right\}$, and let $\gamma_{6}$ be the corresponding hypercube defined by:

$$
\gamma_{6}=\left\{\sum \xi_{i} \varepsilon_{i} \mid 0<\xi_{i}<1, i=1, \ldots, 6\right\} .
$$

Consider the representation of the icosahedral group which permutes the canonical basis of $\mathbb{Z}^{6}$ in the same way as the natural action of this group in $\mathbb{R}^{3}$ permutes 6 suitably chosen of the 12 vectors of the regular icosahedron (see Fig. 2 

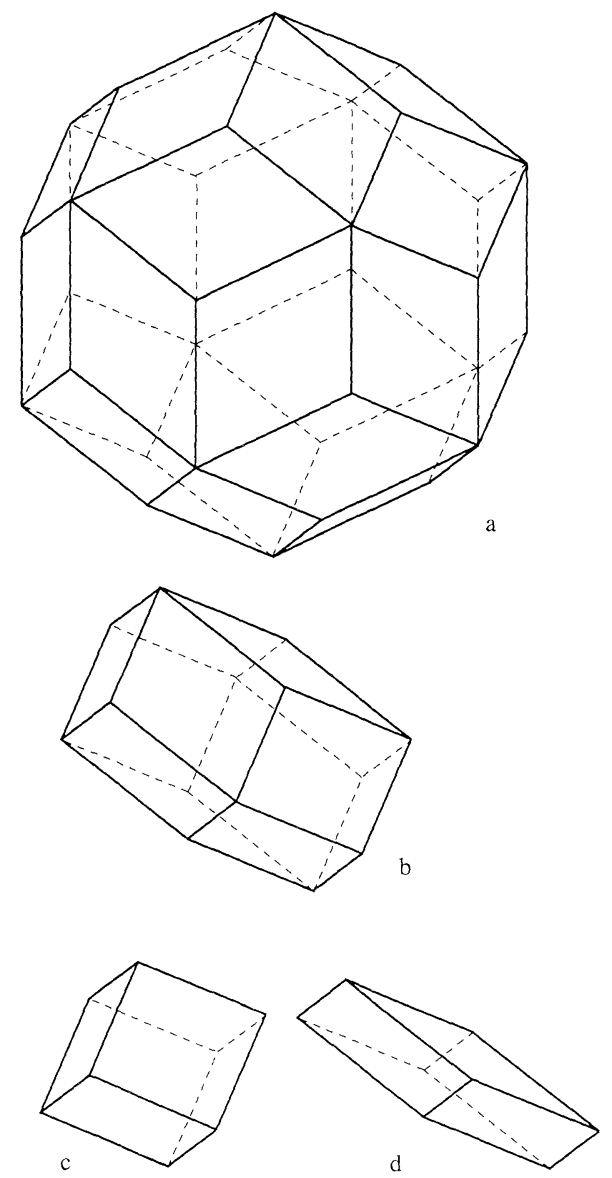

Fig. 1a-d. The polyhedra which are referred to throughout the paper: $\mathbf{a}$ a triacontahedron, $\mathbf{b}$ a dodecahedron, $\mathbf{c}$ a thick rhombohedron, $\mathbf{d}$ a flat rhombohedron
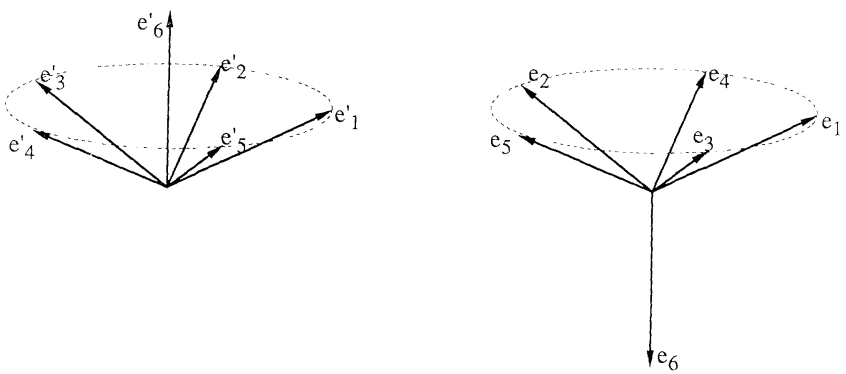

Fig. 2. The projections of the canonical basis vectors of $\mathbb{R}^{6}$ in $\mathbf{E}^{\perp}$ and $\mathbf{E}^{\|}$

for our choice). This group action obviously leaves invariant the lattice $\mathbb{Z}^{6}$, and when decomposing it into irreducible representations, one finds two invariant 3-dimensional subspaces, endowed with the two non-equivalent 3-dimensional representations of the icosahedral group. Now let $\mathbf{E}^{\|}$and $\mathbf{E}^{\perp}$ be these two 3-dimensional subspaces and let us define an open strip in $\mathbb{R}^{n}$ by shifting the cell $\gamma_{6}$ 
along an affine $p$-plane parallel to $\mathbf{E}^{\|}$:

$$
\mathbf{S}_{\alpha}=\mathbf{E}^{\|}+\gamma_{6}+\alpha,
$$

where $\alpha$ is a translation vector in $\mathbb{R}^{6}$.

It is proved in [10] that for translations $\alpha$ such that the boundary of the strip does not contain any point of $\mathbb{Z}^{6}$ (notice that this condition is generic on $\alpha$ ), the strip $\mathbf{S}_{\alpha}$ exactly contains an unique 3-dimensional surface built up of 3-dimensional facets of the lattice $\mathbb{Z}^{6}$, which goes through all the vertices of $\mathbb{Z}^{6}$ falling inside $\mathbf{S}_{\alpha}$. The tilings we are looking for are the projections of these 3-dimensional surfaces on $\mathbf{E}^{\|}$, and the prototiles are the projections of the 3-dimensional facets of the lattice $\mathbb{Z}^{6}$; the twenty different 3 -dimensional facets of $\mathbb{Z}^{6}$ project onto two different rhombohedra, each of which being realized in ten orientations. They are the well-known rhombohedra introduced by Ammann, with identical rhombic facets [with angles $A t n(2)$ and $\pi-A t n(2)]$ (Fig. 1). The lattice $\mathbb{Z}^{6}$ projects on $\mathbf{E}^{\|}$and $\mathbf{E}^{\perp}$ on homogeneous $\mathbb{Z}$-modules and the unit hypercube of $\mathbb{Z}^{6}$ projects onto a rhombic triacontahedron $\mathbf{K}$, a polyhedron with the same 30 rhombic facets, 60 edges and 32 vertices, which presents the full icosahedral symmetry (Fig. 1). Observe that this construction is equivalent to the following: Let $\pi^{\|}$and $\pi^{\perp}$ be the orthogonal projections on $\mathbf{E}^{\|}$and $\mathbf{E}^{\perp}$ respectively, and let:

$$
\mathbf{K}=\pi^{\perp}\left(\gamma_{6}\right)=\pi^{\perp}\left(\mathbf{S}_{0}\right), \quad \mathbf{K}_{\alpha}=\pi^{\perp}\left(\gamma_{6}+\alpha\right)=\pi^{\perp}\left(\mathbf{S}_{\alpha}\right)
$$

(we shall say that $\mathbf{K}_{\alpha}$ is the profile of the strip). The vertices of the tiling corresponding to $\alpha$ are the projections on $\mathbf{E}^{\|}$of the vertices of $\mathbb{Z}^{6}$ which project on $\mathbf{E}^{\perp}$ in the interior of $\mathbf{K}_{\alpha}$, whenever $\alpha$ is such that the boundary of $\mathbf{K}_{\alpha}$ does not intersect the dense $\mathbb{Z}$-module $\pi^{\perp}\left(\mathbb{Z}^{6}\right)$.

Now, if the boundary of $\mathbf{K}_{\alpha}$ intersects $\pi^{\perp}\left(\mathbb{Z}^{6}\right)$, we can again obtain a tiling if we specify, for each pair of opposite facets in the boundary of $\mathbf{K}_{\alpha}$ which contain points of $\pi^{\perp}\left(\mathbb{Z}^{6}\right)$, which one is kept and which one is discarded (we have to be careful if adjacent facets are concerned!) and finally, if in such a situation we project on $\mathbf{E}^{\|}$ all the vertices which fall in the closure of the strip $\mathbf{S}_{\alpha}$, we get the superposition of all possible choices (we shall encounter such situations later in this work).

2.2. This description of the quasiperiodic tilings is very well suited for the study and the classification of the local patterns which appear in the tilings: examine for instance whereall the vertices which are the origin of an edge of a given orientation, say $\pi^{\perp}\left(\varepsilon_{1}\right)$, are: in order for the point $x$ to be such a vertex, we must have altogether in the tiling the vertices $x$ and $x+\pi^{\|}\left(\varepsilon_{1}\right)$, that is, if $x=\pi^{\|}(\xi)$, the two points $x^{\prime}=\pi^{\perp}(\xi)$ and $x^{\prime}+\pi^{\perp}\left(\varepsilon_{1}\right)$ must fall inside the rhombic triacontahedron which defines the tiling or, in other words, the point $x^{\prime}$ must fall in the intersection of the rhombic triacontahedron $\mathbf{K}$ and its translation $\mathbf{K}-\pi^{\perp}\left(\varepsilon_{1}\right)$.

More generally consider the map $\varphi: \pi^{\perp}\left(\mathbb{Z}^{6}\right) \rightarrow \pi^{\|}\left(\mathbb{Z}^{6}\right)$ defined by:

$$
\forall \xi \in \mathbb{Z}^{6}, \quad \varphi\left(\pi^{\perp}(\xi)\right)=\pi^{\|}(\xi) .
$$

Since the kernels $\mathbf{E}^{\|}$and $\mathbf{E}^{\perp}$ of $\pi^{\perp}$ and $\pi^{\|}$intersect the lattice $\mathbb{Z}^{6}$ only at the origin, it is clear that the map $\varphi$ is one to one (however, observe that $\varphi$ is very discontinuous: it maps any bounded set on a discrete set, and thus does not admit any reasonable continuation to $\left.\mathbf{E}^{\perp}\right)$. Then the set of vertices of the tiling is simply $\varphi\left(\mathbf{K}_{\alpha} \cap \pi^{\perp}\left(\mathbb{Z}^{6}\right)\right)$. 
Now, given any local pattern in the tiling attached to an origin $x$, it is clear that the set of vertices in the tiling around which this local pattern is realized is simply the image through $\varphi$ of the intersection of a family of translations of $\mathbf{K}$ (let us call this intersection the existence domain of the pattern) with one copy of $\mathbf{K}$ for each point in the pattern, the corresponding translation in $\mathbf{E}^{\perp}$ being the image through $-\varphi^{-1}$ of the translation which maps $x$ on this point in $\mathbf{E}^{\|}$.

Using the fact that our triacontahedron $\mathbf{K}$ is the projection of an hypercube, it is a simple task to verify the following [9]: (we define $e_{i}=\pi^{\|}\left(\varepsilon_{i}\right)$ and $e_{i}^{\prime}=\pi^{\perp}\left(\varepsilon_{i}\right)$ ).

The existence domains of any edge $e_{i}$ is the rhombic icosahedron spanned by the five vectors $\left\{e_{j}^{\prime}, j \in J\right\}$ such that $J \cup\{i\}=\{1, \ldots, 6\}$. Similarly, the existence domains any facet $\left\{e_{i}, e_{j}\right\}$ is the rhombic dodecahedron spanned by the four complementary vectors $e_{1}^{\prime}$ and finally the existence domains of any rhombohedron $\left\{e_{i}, e_{j}, e_{k}\right\}$ is another rhombohedron, spanned by the complementary vectors, and which is of the same type. Observe that these rhombohedra tile the triacontahedron in several different ways. In fact, one can see that there is only one such tiling up to symmetry, and since this tiling does not present any symmetry, there are 120 different tilings of the triacontahedron by rhombohedra. Finally, observe that each of these existence domains is realized in several positions in the triacontahedron, according to the vertex of the local pattern chosen as its origin.

We are now ready to state the matching rules.

\section{Description of the Matching Rules}

3.1. As explained in the introduction, our goal is to find a set of local rules for packing the tiles, such that the resulting tilings are quasiperiodic. Observe first that the very possibility for the existence of such rules, depends in an essential way on the fact that our triacontahedron is not a generic projection of the hypercube.

More precisely, consider a generic projection of the 6-dimensional hypercubic lattice on a 3-dimensional subspace: then the kernel of the projection is a 3-dimensional subspace which intersects any lattice subspace in general position. This means that the dimension of this intersection is 0 for 1-, 2- or 3-dimensional lattice subspaces, and $m-3$ for an $m$-dimensional subspace, $m>3$.

However, this is not the case for the projection $\pi^{\perp}$ involved in the 3-dimensional Penrose tiling. In fact, we have as a consequence of the icosahedral symmetry a set of relations of the type: $e_{1}^{\prime}-e_{4}^{\prime}$ parallel to $e_{3}^{\prime}-e_{2}^{\prime}$, which can be written:

$$
\left(e_{1}^{\prime}-e_{4}^{\prime}\right) \wedge\left(e_{3}^{\prime}-e_{2}^{\prime}\right)=0 .
$$

By application of the icosahedral group, we obtain fifteen such relations. Each of them means that the corresponding 2-dimensional lattice subspaces spanned by pairs of vectors of the type $\left\{\left(\varepsilon_{1}-\varepsilon_{4}\right),\left(\varepsilon_{3}-\varepsilon_{2}\right)\right\}$ intersects the kernel $\mathbf{E}^{\|}=\left(\pi^{\perp}\right)^{-1}(0)$ along a 1-dimensional subspace (which is not a lattice subspace!), and this is not generic.

Observe that the symmetry implies a maximal nongenericity in the sense that any perturbation of the projection direction breaks at least one of these relations. In other words, these relations force the icosahedral symmetry. 
In the previous section, we have recalled that the existence domains of the tiles are rhombohedra which tile the triacontahedron $\mathbf{K}$. By considering the superposition of all such tilings of $\mathbf{K}$, one gets a cellular decomposition of $\mathbf{K}$ in which each cell corresponds to a possible packing of tiles around a common vertex, and the boundaries of these cells are all the projections of the 2-dimensional facets of the hypercube [9]. As a consequence of the above relations, one can see that many of these projections overlap in $\mathbf{K}$, yielding "flattened" cells of zero volume which correspond to local packings that do not appear in the icosahedrally symmetric tilings but would appear in any tiling defined through a "tilted" $\mathbf{E}$ ". Intuitively, one can hope that by setting rules which forbid these packings, one can force altogether the symmetry and the quasiperiodicity of the permitted tilings, and this is precisely what we are going to do.

On the other hand, the form of the rules we are looking for is simply the matching of decorations of the boundaries of the tiles, which are the 2-dimensional facets. Their existence domains are rhombic dodecahedra, and we have one dodecahedron in $\mathbf{K}$ for each vertex of the facet. We are thus led to examine more precisely these rhombic dodecahedra: they present three orthogonal symmetry planes, two of which intersecting on the long diagonal of the dodecahedron. Consider the eight sectors in which these three planes partition the dodecahedron (Fig. 3). We define the matching rules by setting different decorations for the facets corresponding to different sectors of each dodecahedron and we shall show that this is sufficient to force the symmetry and the quasiperiodicity of all the tilings with matching decorations.

The decoration of each facet is simply a label which indicates in which sector of the relevant dodecahedron falls the image through $\varphi$ of each vertex of this facet. To discuss the choice of these labels (which will be referred to either as the decoration of the facets in $\mathbf{E}^{\|}$or the decoration of the dodecahedra in $\mathbf{E}^{\perp}$ ) observe first that for a given direction of facet, the labelling of the four "parallel" sectors in which fall the image through $\varphi$ of the four vertices of the same facet must be related, in order to correspond to the same decoration for the facet. On the other hand, it is natural to ask for a further condition of symmetry for the decoration, which is simply that the set of decorated tiles of each type should be the same for the ten different orientations of the tiles. This will give constraints, not only on the relation between the decoration of tiles of different orientations, but also on the symmetry of the decoration of each tile.

To explain this point, let us first briefly precise how the symmetry operations are related in $\mathbf{E}^{\|}$and $\mathbf{E}^{\perp}$ : Consider a symmetry operation $\mathbf{g}$ in the high dimensional space $\mathbb{R}^{6}$, preserving $\mathbb{Z}^{6}, \mathbf{E}^{\|}$and $\mathbf{E}^{\perp}$. Then the Shur lemma implies that it takes the form:

$$
\mathbf{g}=\left(\mathbf{g}^{\perp} \otimes \mathrm{id}_{\mathbf{E} \|}\right) \pi^{\perp}+\left(\mathrm{id}_{\mathbf{E}^{\perp}} \otimes \mathbf{g}^{\|}\right) \pi^{\|}
$$

and this formula defines the associated operations in $\mathbf{E}^{\perp}$ and in $\mathbf{E}^{\|}$as well as their relation. Now, observe that it may happen that two sectors of dodecahedra in $\mathbf{K}$ are related in two different ways: first, through a symmetry operation, and second, as being attached to different vertices of the same direction of facet. In this situation, the two decorations derived for the second sector from the decoration of the first must coincide, and this appears as a symmetry constraint on the decoration of the tile itself.

We shall now establish these decorations. 


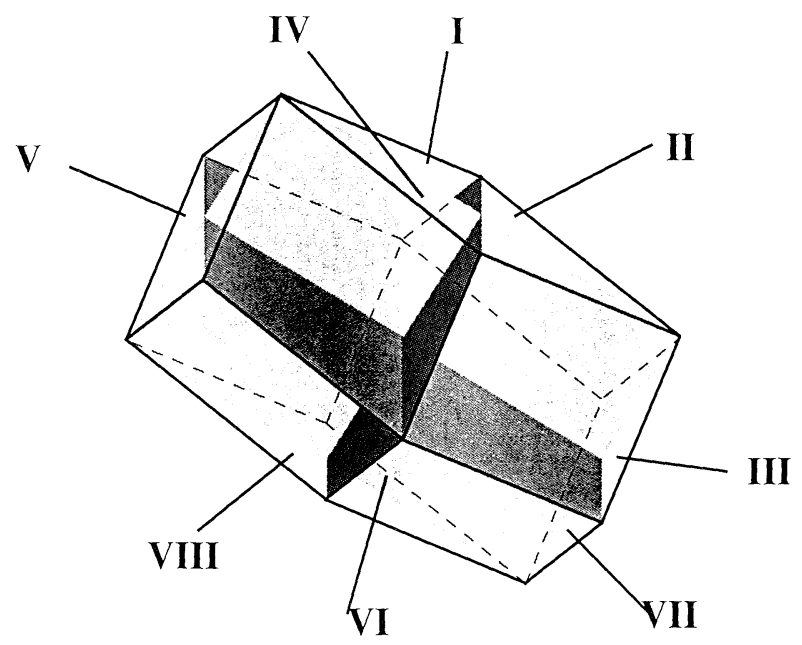

Fig. 3. The eight sectors of the rhombic dodecahedron

3.2. Decoration of the 3-Dimensional Tilings. We have sixty dodecahedra to decorate: one for each vertex of the fifteen directions of facets. Of course, the decoration of dodecahedra corresponding to different vertices of the same facet are to be the same, and we shall use the same notation for parallel sectors in parallel dodecahedra. Let us focus on the symmetry considerations: First, we ask the decorations of dodecahedra corresponding to different orientations to be mapped on each other by the icosahedral group, so that we have only one dodecahedron to decorate. Second, since the icosahedral group is a Coxeter group generated by reflections, we have only to look at the symmetry planes to determine the symmetry of the decoration itself. Consider for instance the dodecahedron $\left\{e_{2}^{\prime}, e_{3}^{\prime}, e_{4}^{\prime}, e_{5}^{\prime}\right\}$ (corresponding to the facet $\left\{e_{1}, e_{6}\right\}$ ) and the symmetry with respect to the plane $\left\{e_{2}^{\prime}, e_{5}^{\prime}\right\}$. It maps the dodecahedron on itself and exchanges the quadruplet of sectors (I, IV, VIII, V) respectively with (II, III, VII, VI). It is easy to see that this symmetry is related in $\mathbf{E}^{\|}$to the symmetry with respect to the short diagonal of the facet, which maps the facet on itself and leaves invariant its origin. This controls the relation between the sectors of the pairs (I, II), (IV, III), (VIII, VII) and (V, VI).

Observe that the origin of the facet being preserved by the operation in $\mathbf{E}^{\|}$is the same property that the dodecahedron being mapped on itself by the corresponding operation in $\mathbf{E}^{\perp}$. Now consider the symmetry plane $\left\{e_{3}^{\prime}, e_{4}^{\prime}\right\}$ : it maps the dodecahedron on another which corresponds to the same facet, attached to the opposite vertex while the related operation in $\mathbf{E}^{\|}$is the symmetry with respect to the long diagonal of the facet, which maps the facet on itself but exchanges the origin with the opposite vertex. Now, this operation exchanges the quadruplet of sectors (I, II, III, IV) respectively with (V, VI, VII, VIII), and this controls the relation between the decoration of sectors of the pairs (I, V), (II, VI), (III, VII) and (IV, VIII). Finally, the symmetry plane $\left\{e_{1}^{\prime}, e_{6}^{\prime}\right\}$ exchanges the quadruplet of sectors (I, II, VI, V) respectively with (IV, III, VII, VIII) in the same dodecahedron, while exchanging in $\mathbf{E}^{\|}$the two sides of the facet $\left\{e_{1}, e_{6}\right\}$. Thus we see that we have only one independent decoration of the facet, which in view of the symmetries involved 

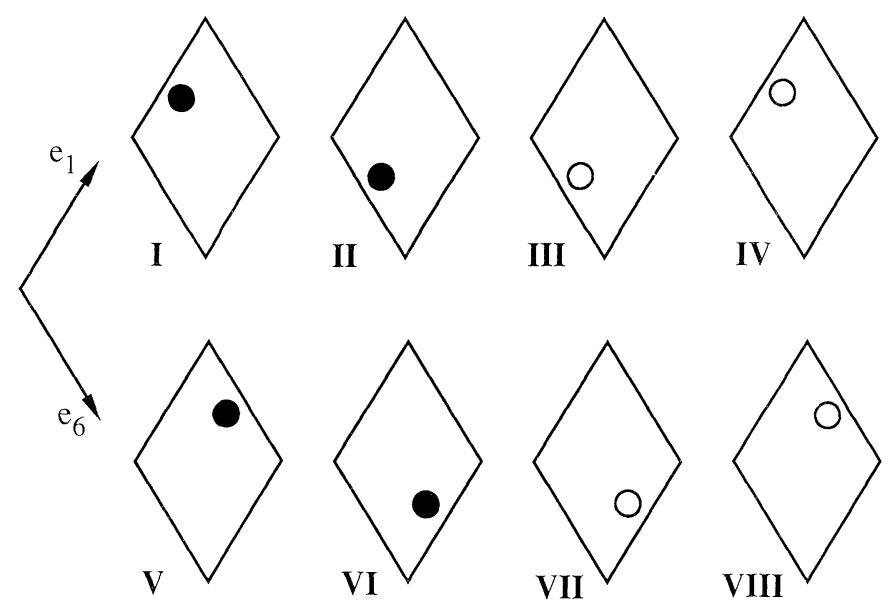

Fig. 4. Decoration of the facets associated with each sector of the dodecahedron

can be chosen as a marking of one quadrant of the rhomb, different on the two sides of the facet (we choose a white disk on a side and a black one on the other) (Fig. 4). Then the rule is to match a white disk with a black one, in order to get the same decorated facet in the two matching rhombohedra.

We have now to examine what are the decorated tiles obtained through this procedure. Consider the tiling defined by the triacontahedron $x^{\prime}+\pi^{\perp}\left(\gamma_{6}\right)$ for any $x^{\prime} \in \mathbf{E}^{\perp}$, and the direction of rhombohedron spanned by $\left\{e_{l}, e_{m}, e_{n}\right\}$. Then, the existence domain of these rhombohedra is the rhombohedron $x^{\prime}+\left\{e_{i}^{\prime}, e_{j}^{\prime}, e_{k}^{\prime}\right\}$, where $(i, j, k, l, m, n)$ is a permutation of $(1, \ldots, 6)$, and we have to examine how this domain is partitioned in subdomains by the partitions of the dodecahedron attached to each of its facets.

Observe first that one of the planes in each of the dodecahedra corresponding to the three facets $x^{\prime}+\left\{e_{i}^{\prime}, e_{j}^{\prime}\right\}$ overlaps with the boundary of the rhombohedron, so that we get only six planes passing through the interior of the rhombohedron from these dodecahedra. For the opposite facets $x^{\prime}+e_{k}^{\prime}+\left\{e_{i}^{\prime}, e_{j}^{\prime}\right\}$, we have to consider the corresponding dodecahedron translated by $-e_{k}^{\prime}$ : since $e_{k}^{\prime}$ belongs to one of the three planes attached to the dodecahedron, we get only two new planes, but one of these two planes overlaps with the boundary of the rhombohedron as previously, so that we see that only the plane orthogonal to the long diagonal give a new partition of our existence domain, yielding three other planes for the three facets. One has to verify that these nine planes divide each thick rhombohedron into forty-two subdomains, and each thin rhombohedron into twenty-four subdomains. The geometry is too involved for a 2-dimensional picture to be readable, so we give here a brief description for both types of rhombohedra.

The geometry admits the diagonal of the rhombohedron as a three-fold axis. The nine planes fall in three families which are: first, three planes containing the long diagonal of the relevant dodecahedron, which intersect along the three-fold axis, and second, two families of three planes orthogonal to these long diagonals. For the thick rhombohedron, each of these last triplets intersects inside the rhombohedron on a point belonging to the three-fold axis, and the intersection of 

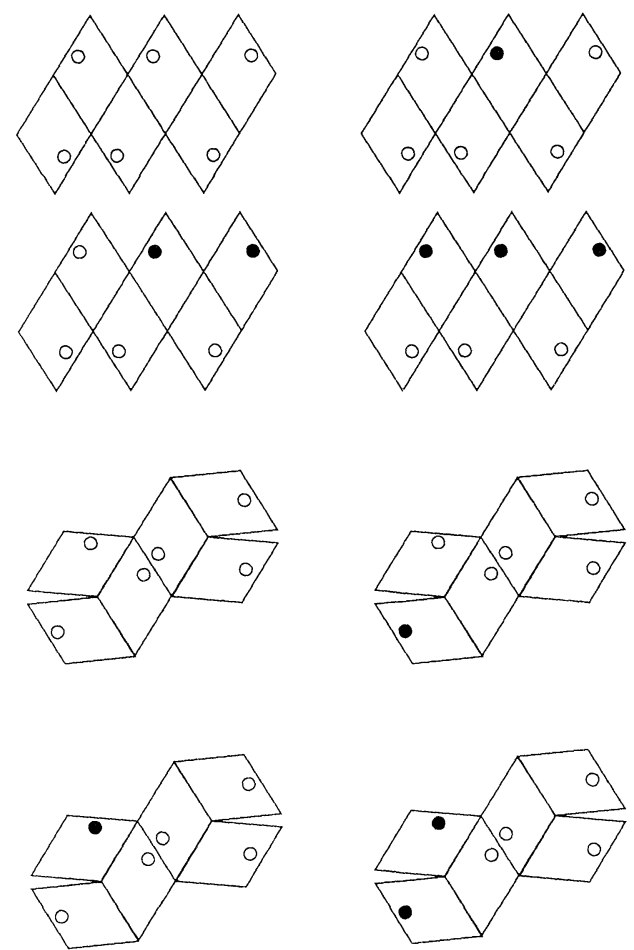

Fig. 5. Four thick and four flat decorated rhombohedra, which generate the whole set

any two planes in these triplets falls on a plane of the first triplet. There is no intersection of two planes belonging to each of the two last triplets inside the rhombohedron, and this determines the whole situation. For the thin rhombohedron, the intersection of any pair of planes in the two last triplets falls outside of the rhombohedron, but we find an intersection for each pair of planes involving one plane from each of the two last triplets.

One can construct the decorated tile corresponding to each of these subdomains by looking in which sector of the relevant dodecahedron falls the origin of each facet, and one is helped in this task by symmetry considerations. When looking at the decorated tiles which are actually different, we see that we have only fourteen of them for the thick rhombohedron, and eight for the thin one. Four of them are depicted on Fig. 5 for each type, and the whole set is generated through the following procedure: For the thin rhombohedron, take the reflection of each of the depicted ones in any of its symmetry planes, and inverse at the same time the color of all the circles. For the thick one, there are two steps. First take the symmetric of each of the depicted rhombohedra with respect to its center, inverting the color of all the circles. There are now eight tiles. Second, take the reflection of each of them in any of its symmetry planes, without changing the circles. Two of the eight are invariant through this operation, so that we get fourteen different decorated thick rhombohedra.

Finally, we get relatively few different tiles. This results from the fact that a third of the new boundaries overlaps with old ones, and this is directly linked with the 
special orientation of the pair $\left(\mathbf{E}^{\|}, \mathbf{E}^{\perp}\right)$. The rest of the paper will be devoted to the proof that the matching of these decorations forces any tiling to be a quasiperiodic tiling. Here is an outline of the proof: Starting with our set of decorated tiles in the ordinary 3-dimensional space, we want to describe any tiling by means of these tiles through a strip projection method with a plane strip, in order to show the quasiperiodicity of the tilings. Of course, we begin by identifying the 3-dimensional space with $\mathbf{E}^{\|}$, and since the projection of $\mathbb{Z}^{6}$ on $\mathbf{E}^{\|}$is one to one, we can lift in $\mathbb{Z}^{6}$ the vertices of the tiling in an essentially unique way (up to a translation in $\mathbb{Z}^{6}$ ). Now, when studying the strips (not necessarily plane) which contain all these vertices, we encounter the following difficulty: there exist infinitely many such strips as soon as there exists one, for we can add some "wiggles" to a given strip without changing the vertices which it contains. Thus, we have to characterize as an equivalence class the set of all the strips associated with a given tiling, and to show that, on account of the matching rules, each class contains a unique plane strip. To achieve this, we first describe in the next section another construction of the Penrose tilings, equivalent to the strip method but in which, loosely speaking, the thickness of the strip is transferred on the vertices. Then the strip reduces to a 3-dimensional surface and we show in the fifth section that the relevant notion of equivalence for these surfaces is that of singular homology in the complementary of a certain "forbidden set" $\Sigma$. In the sixth section, we show that we completely express the matching rules by a suitable choice of the forbidden set. Then we compute the relevant homology group and we show that each class contains a plane, which proves our claim and justifies the choice of the matching rules themselves.

\section{The Cut Description of the Tilings}

4.1. In order to prepare for this proof, let us now describe another construction of these tilings, known as the cut method [10]. This construction appears as an adaptation to the case of tilings of the general description of quasiperiodic sets of points introduced in [11], which is a straightforward generalization of the classical theory of incommensurate structures of Janner and Janssen. The formulation given below, which is simpler than the original version of [10], follows a suggestion of Andreas Dress [12]. The cut construction consists in defining a periodic tiling (referred to as the oblique tiling) in the high dimensional space $\mathbb{R}^{6}$ (with translation group $\mathbb{Z}^{6}$ ) such that the quasiperiodic tilings appear as sections of these periodic tilings through planes parallel to $\mathbf{E}^{\|}$.

The idea is the following: Consider any tiling of $\mathbb{R}^{n}$ and any plane cut through this tiling. Each time the cut is generic, that is, intersects transversely the boundaries of the tiles, the traces of the tiles on the cut make up a covering of the cut, without overlapping nor hole. But this covering is not a tiling in general, since there is no reason for the traces of the tiles to belong to a finite set of shapes. For instance, consider a cut with an irrational slope through the standard square tiling of the plane: since there is no minimal distance between the vertices of the tiling and the cut, there is no minimal length for the segments of the induced covering of the cut, and this entails that there are infinitely many different lengths in this covering. 


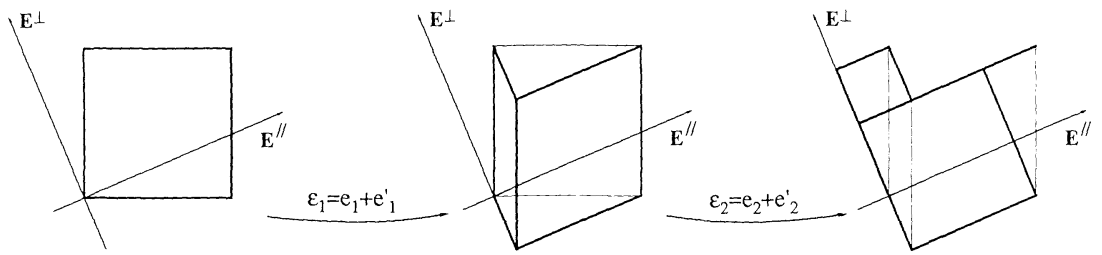

Fig. 6. Construction of the prototiles $C_{I}$ of the oblique tiling, in the lowest dimensions

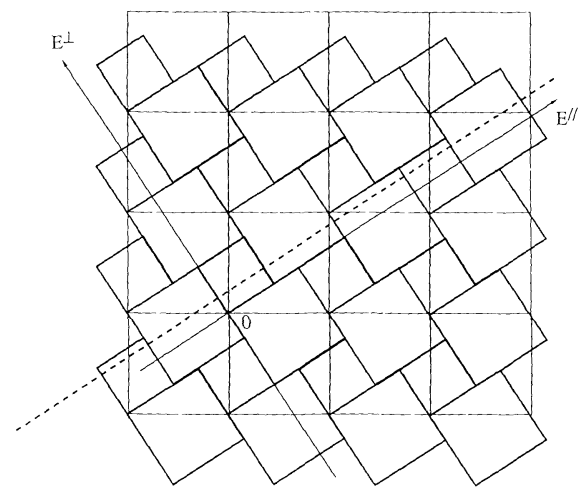

Fig. 7. The oblique tiling in the lowest dimensions

However, it is possible to adapt the shape of the tiles of the tiling of $\mathbb{R}^{n}$ to the orientation of the cut, in order to obtain only a finite number of shapes in the generic cuts: we have to make the boundaries of the tiles parallel to either the cut $\mathbf{E}^{\|}$or its orthogonal $\mathbf{E}^{\perp}$. The construction of such an "oblique tiling" is as follows. Since it is recursive, one can explain it for the plane, without restricting the generality (Fig. 6).

Let us start with the unit square, spanned by the canonical basis $\left(\varepsilon_{1}, \varepsilon_{2}\right)$. We consider the projections $e_{i}=\pi^{\|}\left(\varepsilon_{i}\right)$ and $e_{i}^{\prime}=\pi^{\perp}\left(\varepsilon_{i}\right)$ for $i=1,2$. Observe that $\varepsilon_{1} \wedge \varepsilon_{2}$ $=e_{1} \wedge \varepsilon_{2}+e_{1}^{\prime} \wedge \varepsilon_{2}=1$ (this is the area of the unit square), and that the two exterior products $e_{1} \wedge \varepsilon_{2}$ and $e_{1}^{\prime} \wedge \varepsilon_{2}$ are both positive. This means that the two parallelograms spanned by $\left\{e_{1}, \varepsilon_{2}\right\}$ and $\left\{e_{1}^{\prime}, \varepsilon_{2}\right\}$ do not overlap, so that the union $\left\{e_{1}^{\prime}, \varepsilon_{2}\right\} \cup\left(e_{1}^{\prime}+\left\{e_{1}, \varepsilon_{2}\right\}\right)$ is still a unit cell for the lattice $\mathbb{Z}^{2}$, as depicted on the second part of Fig. 6. Now, let us proceed to the same decomposition for the vector $\varepsilon_{2}$ and each parallelogram. For the same reasons, we get a new unit cell of $\mathbb{Z}^{2}$ made of four subcells spanned by $\left\{e_{1}^{\prime}, e_{2}\right\},\left\{e_{1}^{\prime}, e_{2}^{\prime}\right\},\left\{e_{1}, e_{2}\right\}$ and $\left\{e_{1}, e_{2}^{\prime}\right\}$. But the two subcells spanned by $\left\{e_{1}^{\prime}, e_{2}^{\prime}\right\}$ and $\left\{e_{1}, e_{2}\right\}$ are flat and give a null contribution, so that we obtain finally only two subcells whose union is a fundamental domain of $\mathbb{Z}^{2}$ (last part of Fig. 6). The corresponding tiling of the plane (Fig. 7) is the oblique tiling.

Observe that whatever the order of the decomposition, the resulting tiling is the same. Since each tile is the product of the projection of a basis vector on $\mathbf{E}^{\|}$by the projection of the other on $\mathbf{E}^{\perp}$, it is clear that any cut parallel to $\mathbf{E}^{\|}$which does not intersect the lattice inherits a tiling by means of the two projections $e_{1}$ and $e_{2}$, which is identical to the non-periodic tiling obtained by the projection method. Due to its recursive character, the same argument works in any dimension. For the 
3-dimensional Penrose tilings, it runs as follows: start with the unit hypercube spanned by $\left\{\varepsilon_{1}, \varepsilon_{2}, \varepsilon_{3}, \varepsilon_{4}, \varepsilon_{5}, \varepsilon_{6}\right\}$, decompose $\varepsilon_{1}$ as $e_{1}+e_{1}^{\prime}$ in $\mathbf{E}^{\|}$and $\mathbf{E}^{\perp}$ to obtain a new cell made of the two parallelohedra $\left\{e_{1}, \varepsilon_{2}, \varepsilon_{3}, \varepsilon_{4}, \varepsilon_{5}, \varepsilon_{6}\right\}$ and $\left\{e_{1}^{\prime}, \varepsilon_{2}, \varepsilon_{3}, \varepsilon_{4}, \varepsilon_{5}\right.$, $\left.\varepsilon_{6}\right\}$, and iterate the process with the next basis vector. Since the property of getting a cell of $\mathbb{Z}^{6}$ is preserved at each step, we end with a final cell which is a priori the union of 64 subcells. However, only those which are spanned by three vectors in $\mathbf{E}^{\|}$ and three vectors in $\mathbf{E}^{\perp}$ have a non-zero volume, so that the cell of the oblique tiling is in fact the union of 20 subcells, each of which being the product of an Ammann rhombohedron in $\mathbf{E}^{\|}$by its existence domain in $\mathbf{E}^{\perp}$, in such a way that any cut parallel to $\mathbf{E}^{\|}$which does not intersect the lattice $\mathbb{Z}^{6}$ inherits a 3 -dimensional Penrose tiling.

More precisely, let $M_{p}$ denote the set of $p$-multiindices $\left\{i_{1}, \ldots, i_{p}\right\}$ in $\{1, \ldots, 6\}$, and $\gamma_{I}$ the $p$-dimensional facet of the hypercube $\gamma_{6}$ spanned by the vectors $\varepsilon_{i}$, for $i \in I$ and $I \in M_{p}$. Then the twenty prototiles $C_{I}$ of the oblique tiling take the form:

$$
C_{I}=\pi^{\|}\left(\gamma_{I}\right) \times \pi^{\perp}\left(\gamma_{I^{c}}\right)
$$

with $I \in M_{3}$, and where $I^{c}$ is the complementary of $I$ in $\{1, \ldots, 6\}$.

4.2. Although the definitions of the periodic oblique tiling and of the prototiles $C_{I}$ are very simple, the overall geometry is rather involved. Here are some details on the structure of the boundaries of the tiles $C_{I}+\xi$. Let us denote $\partial C_{I}$ the boundary of $C_{I}$ : It is a cellular complex of dimension 5 whose cells are $(6-q)$-dimensional facets of the hypercube $C_{I}$, for $q>0$. Now, the 5-dimensional cells of $\partial C_{I}$ fall into two classes: those which are the product of a 3-dimensional facet $\pi^{\|}\left(\gamma_{I}\right)$ in $\mathbf{E}^{\|}$by a 2-dimensional facet belonging to $\partial\left(\pi^{\perp}\left(\gamma_{I^{c}}\right)\right)$ in $\mathbf{E}^{\perp}$ (we shall say that these cells are parallel to $\mathbf{E}^{\|}$and denote their union by $\partial_{\|} C_{I}$ ) and those which are the product of a 2-dimensional facet of $\partial\left(\pi^{\|}\left(\gamma_{I}\right)\right)$ in $\mathbf{E}^{\|}$by a 3-dimensional facet $\pi^{\perp}\left(\gamma_{I^{c}}\right)$ in $\mathbf{E}^{\perp}$ (we shall say that these cells are parallel to $\mathbf{E}^{\perp}$ and denote their union by $\partial_{\perp} C_{I}$ ). Accordingly, the union of the boundaries of all the tiles in the periodic lattice falls into two subsets $\mathbf{B}_{\perp}$ and $\mathbf{B}_{\|}$defined by:

$$
\mathbf{B}_{\perp}=\bigcup_{M_{3} \times \mathbb{Z}^{6}}\left(\partial_{\perp} C_{I}+\xi\right), \quad \mathbf{B}_{\|}=\bigcup_{M_{3} \times \mathbb{Z}^{6}}\left(\partial_{\|} C_{I}+\xi\right) .
$$

One can verify the following geometric properties: (see Figs. 6 and 7).

- The set of cuts which do not intersect $\mathbf{B}_{\|}$is generic, and each such cut defines a quasiperiodic tiling.

- The intersection of such a generic cut with $\mathbf{B}_{\perp}$ is the set of the boundaries of tiles in this tiling.

More generally, we can describe the set $\mathbf{B}_{\perp}$ and $\mathbf{B}_{\|}$by their intersections with the fibers of the projection $\pi^{\perp}$ : as already mentioned, whenever the fiber is generic, its intersection with $\mathbf{B}_{\|}$is empty. Let us examine the situation when the fiber intersects a cell of maximal dimension in $\partial_{\|} C_{I}+\xi$, and suppose $\xi=0$ for simplicity.

Recall that the prototile $C_{I}$ has the form of a product $\pi^{\|}\left(\gamma_{I}\right) \times \pi^{\perp}\left(\gamma_{I^{c}}\right)$, and that the boundary $\partial_{\|} C_{I}$ is $\pi^{\|}\left(\gamma_{I}\right) \times \partial\left(\pi^{\perp}\left(\gamma_{I}\right)\right)$, in such a way that each cell in $\partial_{\|} C_{I}$ takes the form of a product $\pi^{\|}\left(\gamma_{I}\right) \times \pi^{\perp}\left(\gamma_{J}\right)$, where $J \in M_{2}$ and $J \subset I^{c}$, which means that $\gamma_{J}$ is a 2-facet of the boundary of the cube $\gamma_{I^{c}}$. Then the intersection is the product of a point in $\pi^{\perp}\left(\gamma_{J}\right)$ by $\pi^{\|}\left(\gamma_{I}\right)$. Now observe that the same $\pi^{\perp}\left(\gamma_{J}\right)$ appears in all the boundaries $\partial_{\|} C_{L}$ with $J \subset L^{c}$. This entails that the given fiber intersects these several boundaries as soon as it intersects one of them, and if one takes into account the 
translations, one can see that the complete intersection is simply the product of a point in $\pi^{\perp}\left(\gamma_{J}\right)$ by $\pi^{\|}\left(\gamma_{J^{c}}\right)$, thus is the projection of the 4-facet $\gamma_{J^{c}}$ of the hypercube. Moreover, $\pi^{\| \prime}\left(\gamma_{J^{c}}\right)$ is covered twice by the boundaries of the type $\partial_{\|} C_{I}+\xi$, namely from both sides of the affine support of the product $\mathbf{E}^{\|} \times \pi^{\perp}\left(\gamma_{J}\right)$. (Notice that we could get rid of these translations in a systematic way, simply by considering the cellular decomposition of the torus $\mathbb{T}^{6}=\mathbb{R}^{6} / \mathbb{Z}^{6}$ induced by the periodic tiling rather than the decomposition of $\mathbb{R}^{6}$ itself. However, it is likely that this formally better description would not help the reader in visualizing the situation.)

Finally we get in the fiber the projection of a 4-dimensional facet of the unit hypercube, (this projection is a rhombic dodecahedron) together with all its possible tilings by the projection of 3-dimensional facets. Of course, we do not assert that this situation occurs only one time in a given fiber: it may happen for symmetry reasons that a fiber intersects $\mathbf{B}_{\|}$in many different places, and the previous considerations hold for each intersection. Consider now the intersection of the same fiber with $\mathbf{B}_{\perp}$ : we have only to observe that we get in the fiber a boundary of a tile of the type $\partial\left(\pi^{\|}\left(\gamma_{I}\right)\right)$ for each cell of $\mathbf{B}_{\|}$contained in the fiber, which correspond to the 3-subsets $I \supset J$. Loosely speaking, such a fiber is to be considered as a "bifurcation point" which contains the superposition of the two regular tilings of a suitable compact neighborhood of the intersection, arising from a small displacement of the fiber on each side of the hyperplane $\mathbf{E}^{\|} \times \pi^{\perp}\left(\gamma_{J}\right)$, and which differ precisely by the way the projection through $\pi^{\| \prime}$ of a 4-dimensional facet of the hypercube is tiled.

The whole discussion can be carried in higher codimensions. Let us simply state the following: If the fiber intersects $\mathbf{B}_{\|}$on a $(3-q)$-dimensional cell, then it intersects at the same time all the cells that are contained in the projection of a $(3+q)$-dimensional facet of the unit hypercube, and the corresponding intersection with $\mathbf{B}_{\perp}$ appears as a "bifurcation point" which contains all the possible ways of tiling the projection of a $(3+q)$-dimensional facet of the hypercube.

As a final remark on this description, observe that one can develop the same analysis using the fibers of $\pi^{\|}$rather than $\pi^{\perp}$. Then the generic fibers intersect $\mathbf{B}_{\|}$ along the boundaries of a tiling by means of the existence domains of the previous tiles. To sum up, let us consider the geometry in the fiber $\pi^{\|-1}(x)$ in the neighborhood of $x$ when $x$ runs over a generic cut bearing a quasiperiodic tiling: For $x$ in the interior of any tile, we see in the fiber the (boundaries of the) existence domain of this tile. When $x$ comes on a 2-dimensional facet of the tiling, we see in the fiber the existence domain of this 2-dimensional facet, which is a dodecahedron, together with its two tilings by means of existence domains of tiles (there are two such tilings, simply because there are two ways of approaching a 2-facet: from each side). This superposition yields a cellular decomposition of the existence domain of the 2-facet, in which each cell corresponds to a possible pair of tiles sharing a 2-facet, and the situation actually realized around $x$ in the cut corresponds to the cell in which $x$ lies in the fiber. In higher codimensions, the geometry is very similar: if $x$ comes on a $(3-q)$-dimensional facet of the quasiperiodic tiling, then we get in the fiber $\pi^{\|-1}(x)$ the existence domain of this $3-q$ facet with all its possible tilings, and each cell of the induced decompositions corresponds to a possible environment of this type of $3-q$ facet in the tiling. In particular, we recover in the case $q=3$ the classification of local packings of tiles around a common vertex which was mentioned in Sect. 3.1 of this paper. 


\section{The Homology Class of a Cut}

5.1. Up to now, we have used plane cuts, but we shall need a generalization of this point of view. Observe that in the previous description the cut plays a double role. On one hand, it works as a means of selecting vertices or tiles, and on the other, it is the space on which the tiling is actually built. We shall now distinguish between these two roles. Let us fix once and for all $\mathbf{E}^{\|}$as the space on which the tiling is built by projection of vertices of $\mathbb{Z}^{6}$ through $\pi^{\|}$. Now one can use 3-dimensional surfaces more general than planes to select the vertices to be projected.

To explain the suitable extension of this notion, let us first deal with the problem of selecting vertices of $\mathbb{Z}^{6}$. Consider the set:

$$
\Sigma_{0}=\left\{\bigcup_{\mathbb{Z}^{6}}\left(\mathbf{E}^{\perp}+\xi\right)\right\} \cap \mathbf{B}_{\perp} .
$$

It consists in a set of copies of $\mathbf{K}$ (recall that the triacontahedron $\mathbf{K}$ is the projection through $\pi^{\perp}$ of the unit hypercube of $\mathbb{R}^{6}$ and is a 3-dimensional cellular complex) translated at each vertex of $\mathbb{Z}^{6}$. Let us denote $\partial^{-1} \sigma_{\xi}$ the set $\mathbf{K}+\xi$. It is trivial that a cut along the plane $\mathbf{E}^{\|}+\alpha$ selects by intersection with $\Sigma_{0}$ exactly the same vertices as the strip $\mathbf{K}_{\alpha}+\mathbf{E}^{\|}$, and this is the very relation between the strip projection and the cut descriptions of our tilings. Now for a general 3-dimensional surface $\mathbf{F}$, we can consider the family (indexed by $\xi \in \mathbb{Z}^{6}$ ) of intersection indices $\left(\mathbf{F} \mid \partial^{-1} \sigma_{\xi}\right)$. If we set the orientations such that for $\mathbf{F}$ being a generic plane cut these intersection indices are 0 or +1 , then for a general $\mathbf{F}$ we define the selected vertices as those $\xi$ for which the intersection index $\left(\mathbf{F} \mid \partial^{-1} \sigma_{\xi}\right)$ is +1 , and we shall consider only surfaces such that all the intersection indices are 0 or +1 . Notice that the intersection index is well defined only when $\mathbf{F}$ does not intersect the boundary $\sigma_{\xi}$ of $\partial^{-1} \sigma_{\xi}$. We shall only consider surfaces $\mathbf{F}$ fulfilling this condition which generalizes the genericity condition we have used up to now.

5.2. As is well known from elementary algebraic topology, the notion of intersection index falls to homology as a pairing between homology groups. We shall use plain singular homology with compact supports, considering the intersection index as a pairing between the homology group $H_{3}\left(\mathbb{R}^{6}-\Sigma_{0}\right)$ and the relative homology group $H_{3}\left(\mathbb{R}^{6}, \Sigma_{0}\right)$. More precisely, in order to avoid inessential difficulties arising from infinite rank homology groups or from the precautions needed with duality theorems for homology with closed supports, we define a "large" convex bounded open set $\mathbb{U}$ in $\mathbb{R}^{6}$ and we consider instead of the whole periodic tiling (and the attached geometrical objects such as $\mathbf{B}_{\|}$or $\Sigma_{0}$ ), its intersection with $\mathbb{U}$. Actually, far from being a technical point, the introduction of such an $\mathbb{U}$ sets the natural context for our main proof, which is of a local nature.

Thus we are dealing in our demonstrations only with finite cellular complexes, and it is obviously possible to choose $\mathbb{U}$ such that any cell, tile, facet etc., which intersects $\mathbb{U}$ is contained in its closure, each time this is necessary for our argument to work.

5.3. Let us now prove three lemmas:

Lemma 1. For $p<n-1$, the natural homomorphism

is an isomorphism.

$$
H_{n-p}\left(\mathbb{R}^{n}, \Sigma_{0} \cap \mathbb{U}\right) \rightarrow H_{n-p-1}\left(\Sigma_{0} \cap \mathbb{U}\right)
$$


This follows immediately from the exact sequence for relative homology. Consider this exact sequence:

$$
\cdots \rightarrow H_{n-p}\left(\mathbb{R}^{n}\right) \rightarrow H_{n-p}\left(\mathbb{R}^{n}, \Sigma_{0} \cap \mathbb{U}\right) \rightarrow H_{n-p-1}\left(\Sigma_{0} \cap \mathbb{U}\right) \rightarrow H_{n-p-1}\left(\mathbb{R}^{n}\right) \rightarrow \cdots
$$

If $n-p-1$ is $>0$, the groups $H_{n-p}\left(\mathbb{R}^{n}\right)$ and $H_{n-p-1}\left(\mathbb{R}^{n}\right)$ are both zero and the exactness implies that the central arrow is an isomorphism.

This is of course very classical and we explicit it mainly for stating the two following remarks: First, the symbol $\partial^{-1}$ in the notation $\partial^{-1} \sigma_{\xi}$ refers to a welldefined operation, which is the inverse of this isomorphism. Second, this lemma is false in the case $n=2, p=1$. So the reader should forget from now on about Fig. 7, which is becoming misleading for the next developments.

Finally, observe that this lemma is independent of the topology of the set $\Sigma_{0}$. We shall use it without reference for other sets $\Sigma$.

Lemma 2. The set $\left\{\sigma_{\xi} \mid \xi \in \mathbb{Z}^{6} \cap \mathbb{U}\right\}$ is a basis of $H_{2}\left(\Sigma_{0} \cap \mathbb{U}\right)$.

Recall that $\sigma_{\xi}=\partial \mathbf{K}+\xi$, so $\sigma_{\xi}$ is topologically a 2-dimensional sphere, and $H_{2}\left(\sigma_{\xi}\right)=\mathbb{Z}$. Then the lemma is trivial, since $\Sigma_{0}$ is the disjoint union of the triacontahedra $\sigma_{\xi}$. This is very elementary but important for us, since it shows that if we consider a cut without boundary (think of any cut across $\mathbb{U}$ and close it outside $\mathbb{U})$ then its homology class in $H_{3}\left(\mathbb{R}^{6}-\left(\Sigma_{0} \cap \mathbb{U}\right)\right)$ completely describes the points which it selects. In fact, we shall be mainly concerned in proving that some special sets of classes are basis of relevant homology groups.

5.4. This conclude our considerations about selecting vertices with homology classes. Now the natural question arises: Is it possible to enlarge the "forbidden set" $\Sigma_{0}$ to a set $\Sigma$ such that classes in $H_{3}\left(\mathbb{R}^{6}-(\Sigma \cap \mathbb{U})\right)$ select not only points, but whole local patterns, for instance tiles? This is in fact the case, as shows our next lemma:

Lemma 3. Define $\Sigma_{1}$ as $\mathbf{B}_{\|}$. Then the set $\left\{\partial_{\|} C_{I}+\xi \mid I \in M_{3}, \xi \in \mathbb{Z}^{6} \cap \mathbb{U}\right\}$ is a basis of $\mathrm{H}_{2}\left(\Sigma_{1} \cap \mathbb{U}\right)$.

This will easily follow from the exactness of the Mayer-Vietoris sequence. Observe first that $\partial_{\|} C_{I}$ admits the boundary of a rhombohedron as a deformation retract (through a retraction along $\mathbf{E}^{\|}$), and this proves that $H_{2}\left(\partial_{\|} C_{I}\right)=\mathbb{Z}$. Now one can build $\Sigma_{1} \cap \mathbb{U}$ by successive additions of pieces $\partial_{\|} C_{I}+\xi$, and since $\mathbb{U}$ is convex it is clear that one can proceed in such a way that each new piece is glued on the periphery of the construction, and thus, is glued along a contractible subset. Let us compute recursively the homology. Define $\Sigma$ as the set obtained at the $m^{\text {th }}$ step. Then add a new piece $\partial_{\|} C_{I}+\xi$ to get $\Sigma^{\prime}$. The corresponding Mayer-Vietoris sequence reads:

$$
\cdots \rightarrow H_{2}\left(\Sigma \cap\left(\partial_{\|} C_{I}+\xi\right)\right) \rightarrow H_{2}(\Sigma) \oplus H_{2}\left(\partial_{\|} C_{I}+\xi\right) \rightarrow H_{2}\left(\Sigma^{\prime}\right) \rightarrow H_{1}\left(\Sigma \cap\left(\partial_{\|} C_{I}+\xi\right)\right) \rightarrow \cdots
$$

Since the intersection is contractible, the two extreme groups are zero, and the central arrow is an isomorphism. We thus see that

$$
H_{2}\left(\Sigma_{1} \cap \mathbb{U}\right)=\underset{M_{3} \times\left(\mathbb{Z}^{6} \cap \mathbb{U}\right)}{\oplus} H_{2}\left(\partial_{\|} C_{I}+\xi\right),
$$

which proves the lemma. 
The meaning of this result is that any class in $H_{3}\left(\mathbb{R}^{6}-\left(\Sigma_{1} \cap \mathbb{U}\right)\right)$ selects whole tiles, and that any set of tiles can be selected in this way. In fact, Lemma 1 implies that $H_{2}\left(\Sigma_{1} \cap \mathbb{U}\right)$ is isomorphic to $H_{3}\left(\mathbb{R}^{6}, \Sigma_{1} \cap \mathbb{U}\right)$, and duality by intersection that this last group is isomorphic to $H_{3}\left(\mathbb{R}^{6}-\left(\Sigma_{1} \cap \mathbb{U}\right)\right)$. Of course, these isomorphisms map basis on basis, in such a way that we get a basis of $H_{3}\left(\mathbb{R}^{6}-\left(\Sigma_{1} \cap \mathbb{U}\right)\right)$ made of classes which select only one tile. Now, an arbitrary set of tile is selected by a class obtained as the sum of the corresponding basis classes, with coefficients +1 .

Observe that since $\Sigma_{0} \subset \Sigma_{1}$, we can consider the classes of $\sigma_{\xi}$ in $\Sigma_{1}$. It is clear that these classes still generate the group $H_{2}\left(\Sigma_{1} \cap \mathbb{U}\right)$, but they are no longer independent: the relations among them precisely express that any cycle in $H_{3}\left(\mathbb{R}^{6}-\left(\Sigma_{1} \cap \mathbb{U}\right)\right)$ necessarily crosses simultaneously the eight classes associated with the vertices of a rhombohedron.

Let us briefly examine how the classes can be realized which span $\partial^{-1} H_{2}\left(\partial_{\|} C_{I}+\xi\right)$. Recall that the fibers $\pi^{\perp-1}\left(\pi^{\perp}(\zeta)\right)$ intersect $\Sigma_{1}$ in a neighborhood of $\zeta$ along a rhombic triacontahedron tiled in all possible ways by rhombohedra. Now for each boundary of these rhombohedra, there is a pair $(I, \xi)$ such that it is a deformation retract of $\partial_{\|} C_{I}+\xi$. Then the class of this boundary spans the group $\mathrm{H}_{2}\left(\partial_{\|} \mathrm{C}_{I}+\xi\right)$, and we can take the whole rhombohedron as the generator of $\partial^{-1} H_{2}\left(\partial_{\|} C_{I}+\xi\right)$. Observe that this realizes the same class in the eight different triacontahedra corresponding to the eight vertices of each facet, and that the direct sum of all the groups $\partial^{-1} H_{2}\left(\partial_{\|} C_{I}+\xi\right)$ is $H_{3}\left(\mathbb{R}^{6}, \Sigma_{1} \cap \mathbb{U}\right)$. Finally, observe that the constraints resulting from the enlargement of $\Sigma_{0}$ to $\Sigma_{1}$ are rather weak: we get tiles, but we still have very far to go to get tilings, since any set of tiles (including interpenetrating tiles) can be obtained in this way.

This situation changes drastically when one takes the matching rules into account.

\section{The Geometry of the Oblique Tiling}

6.1. In Sect. 4.2, we have given a description of $\Sigma_{1}=\mathbf{B}_{\|}$through its intersections with the fibers of the two projections $\pi^{\|}$and $\pi^{\perp}$. In the fibers of $\pi^{\|}$, the description was global (we get the boundaries of a tiling by existence domains in the generic fibers, and the superposition of such tilings in non-generic fibers) but in the nongeneric fibers of $\pi^{\perp}$ the description was only local (we get projections of facets of the hypercube, and the dimension of the facet depends on the degree of non-genericity of the fiber). We then mentioned the possibility of finding many different projections in the same fiber, and we shall now give more precisions on this question.

Let us parametrize the fibers by points in $\mathbf{E}^{\perp}$. We study the intersection $\Sigma_{1}$ $\cap \pi^{\perp-1}\left(x^{\prime}\right), x^{\prime} \in \mathbf{E}^{\perp}$, and we already know that such intersections are made of rhombic dodecahedra. Recall that $\Sigma_{1}$ is the union of all $\partial_{\|} C_{I}+\xi$, so that

$$
\pi^{\perp}\left(\Sigma_{1}\right)=\pi^{\perp}\left(\mathbb{Z}^{6}\right)+\bigcup_{I \in M_{3}} \pi^{\perp}\left(\partial_{\|} C_{I}\right)
$$

Observe that $\pi^{\perp}\left(\partial_{\|} C_{I}\right)=\partial \pi^{\perp}\left(C_{I}\right)$. This last set consists in a union of 2-dimensional facets $\pi^{\perp}\left(\gamma_{J}\right)$ (they are the facets $\left\{e_{i}^{\prime}, e_{j}^{\prime}\right\}$ with $J=\{i, j\}$ ), which come by pairs associated with pairs of opposite facets of the cube $\gamma_{I^{c}}$. However, since the 
translation which maps each facet on the opposite falls in $\pi^{\perp}\left(\mathbb{Z}^{6}\right)$, it is sufficient to keep only one of them and we can write:

$$
\pi^{\perp}\left(\Sigma_{1}\right)=\pi^{\perp}\left(\mathbb{Z}^{6}\right)+\bigcup_{J \in M_{2}} \pi^{\perp}\left(\gamma_{J}\right)
$$

We see that the facets corresponding to a given $J$ cover whole planes through translations in $\pi^{\perp}\left(\mathbb{Z}^{6}\right)$, in such a way that $\pi^{\perp}\left(\Sigma_{1}\right)$ is made of fifteen dense families of planes parallel to the fifteen directions of facets. Suppose first that $x^{\prime}$ belongs to only one plane (this the lower codimension case). Now the intersection $\Sigma_{1}$ $\cap \pi^{\perp-1}\left(x^{\prime}\right)$ contains all the rhombohedra $\pi^{\|}\left(\gamma_{I}+\xi\right)$ such that the boundary of the rhombohedron $\pi^{\perp}\left(\gamma_{I^{c}}\right)+\pi^{\perp}(\xi)$ contains $x^{\prime}$, and our next step is to show that this intersection can be obtained through a strip projection.

In fact, consider the boundary $x^{\prime}+\partial \pi^{\perp}\left(\gamma_{I^{c}}\right)$. Then it is immediate that the origins of the rhombohedra $\pi^{\|}\left(\gamma_{I}+\xi\right)$ in $\Sigma_{1} \cap \pi^{\perp-1}\left(x^{\prime}\right)$ are precisely $\varphi\left(x^{\prime}+\partial \pi^{\perp}\left(\gamma_{I^{c}}\right)\right)$, and we have to make this construction for all $I \in M_{3}$. However, since we are dealing with the lower codimensional case, only one pair of facets intersects $\pi^{\perp}\left(\mathbb{Z}^{6}\right)$, namely $x^{\prime}+\pi^{\perp}\left(\gamma_{J}\right)$ and its opposite in $\partial \pi^{\perp}\left(\gamma_{I^{c}}\right)$, in such a way that we have only to consider the four directions $I$ with $I^{c}=J \cup\{i\}, i \notin J$, and for these $I$, only the two facets parallel to $\pi^{\perp}\left(\gamma_{J}\right)$. To each of the vertices selected by this special strip, we attach a rhombohedron $\pi^{\|}\left(\gamma_{I}\right)$. In order to reorganize this set, it is useful to build the strip which selects all the vertices of the rhombohedra of $\Sigma_{1} \cap \pi^{\perp-1}\left(x^{\prime}\right)$ : we simply have to associate to each facet $\pi^{\perp}\left(\gamma_{J}\right)$ which selects the origin of a rhombohedron, the octuplet of facets which select all its vertices. We leave it to the reader to verify that one finally obtains a set of sixteen parallel facets $x^{\prime}+\pi^{\perp}\left(\gamma_{J}\right)+v_{i}$, where the sixteen vectors $v_{i}$ are the vertices of the rhombic dodecahedron $\pi^{\perp}\left(\gamma_{J^{c}}\right)$.

Thus we find a double description of $\Sigma_{1} \cap \pi^{\perp-1}\left(x^{\prime}\right)$. On one hand, it is "nearly" a set of rhombohedra extracted from a 3-dimensional Penrose tiling. More precisely, observe that the set of sixteen facets fits exactly in the close rhombic triacontahedron $x^{\prime}+\pi^{\perp}\left(\gamma_{6}\right)$. However, since our strip involves two opposite facets of the triacontrahedron, namely:

$$
x^{\prime}+\pi^{\perp}\left(\gamma_{J}\right) \quad \text { and } \quad x^{\prime}+\pi^{\perp}\left(\gamma_{J}\right)+\sum_{i \in J^{c}} e_{i}^{\prime},
$$

we get the kind of "overtiling" which was mentioned at the end of Sect. 2.1.

On the other hand, it is a distribution of rhombic dodecahedra. These dodecahedra are parallel to $\pi^{\|}\left(\gamma_{J^{c}}\right)$, and their origins are selected by the strip $x^{\prime}+\pi^{\perp}\left(\gamma_{J}\right)+\mathbf{E}^{\|}$. We thus get a quasiperiodic set of dodecahedra in the plane $x^{\prime}+\left\{\sum \lambda_{i} e_{i} \mid i \in J, \lambda_{i} \in \mathbb{R}\right\}$. Notice that for each of these dodecahedra we have the superposition of its two tilings by rhombohedra, and this is the "overtiling" mentioned above. We shall not need a complete description of this quasiperiodic set. Observe only that a facet is small enough for this set being not connected: some of the dodecahedra form connected systems, but there are never more than a finite number of them in each connected component.

Finally, observe that when $x^{\prime}$ belong to an intersection of different planes in $\pi^{\perp}\left(\mathbb{Z}^{6}\right)$, we get essentially a union of sets of the type we have just described. Let us make two remarks about these results. First, the fact that the intersection $\Sigma_{1}$ $\cap \pi^{\perp-1}\left(x^{\prime}\right)$ is made of a whole quasiperiodic set (extending to infinity) of polyhedra, rather than being bounded is a direct consequence of the orientation of the pair 
$\left(\mathbf{E}^{\perp}, \mathbf{E}^{\|}\right)$being non-generic, and corresponds to the possibility for the existence of matching rules forcing quasiperiodicity, as we shall see below. Second, the presence of such an infinite set in the fiber is not by itself a constraint on the system of tiles a cut can select, as already mentioned in Sect. 5.3. Intuitively, one can see that a 3-dimensional surface can turn around the connected components of $\Sigma_{1}$ and reach any tile $C_{I}+\xi$ of the oblique tiling.

6.2. The next step is to express our matching rules in the context of the cut description. Let us first define the related periodic tiling of $\mathbb{R}^{6}$. Its prototiles are simply the products of the (decorated) tiles in $\mathbf{E}^{\|}$by their existence subdomains in $\mathbf{E}^{\perp}$. This amounts only to cutting each $C_{I}$ in fortx-two or twenty-four pieces so that it is plain that we get a periodic tiling in $\mathbb{R}^{6}$.

As in the previous case, we can define the subsets $\mathbf{B}_{\|}$and $\mathbf{B}_{\perp}$ of the set of all the boundaries of tiles in this new periodic tiling. We are specifically interested in the set $\mathbf{B}_{\|}$, and let us call this set $\Sigma_{2}$. Observe that we have the relations $\Sigma_{1} \subset \Sigma_{2}$ and $\pi^{\perp}\left(\Sigma_{1}\right)=\pi^{\perp}\left(\Sigma_{2}\right)$. The first one is trivial, since all the boundaries of tiles of the previous tiling appear in the new one. The second merely expresses that any tiling associated with a generic plane cut can be decorated, which is already known from the very definition of the decoration.

One can describe $\Sigma_{2}$ through its intersections with the fibers of $\pi^{\perp}$ or $\pi^{\|}$. We shall work out the description of $\Sigma_{2} \cap \pi^{\perp-1}\left(x^{\prime}\right)$ for all $x^{\prime} \in \mathbf{E}^{\perp}$, and let us simply remark here that the intersection of a generic fiber of $\pi^{\|}$with $\Sigma_{2}$ is the set of boundaries of a tiling of the fiber by means of the existence subdomains of decorated tiles. For non-generic fibers, one gets as usual a superposition of such tilings and one can see these fibers as "bifurcation points."

Now, it is most important to observe that any cut which does not intersect $\Sigma_{2}$ exactly selects decorated tiles. To see this, we have only to make a local verification. In fact, consider a cut which does not intersect $\Sigma_{1}$ (and thus selects whole tiles) but intersects one of the new internal partitions of a tile $C_{I}+\xi$. Draw a line on the cut, going through this intersection, and examine what happens in $\mathbf{E}^{\|}$for the projection of the selected tiles: when one follows the projection of the line, one enters in the tile $\pi^{\|}\left(\gamma_{I}+\xi\right)$ through a facet bearing the decoration corresponding to a certain subdomain of $C_{I}+\xi$, and one goes out through a facet bearing the decoration of another subdomain. But the resulting tile is not one of our decorated tiles. We can thus conclude that defining the set $\Sigma_{2}$ as the new "forbidden set" is the exact expression of our matching rules in the context of the cut method.

\section{The Main Theorem}

7.1. We turn now to the description of the set $\Sigma_{2}$. The following lemma is the geometric key of our theorem. Consider a point $x^{\prime} \in \mathbf{E}^{\perp}$ belonging to only one plane of $\pi^{\perp}\left(\Sigma_{1}\right)$. Then the following holds:

Lemma 4. The set $\Sigma_{2} \cap \pi^{\perp-1}\left(x^{\prime}\right)$ admits a 2-dimensional plane as a deformation retract.

Proof. Since the matter is becoming rather technical, we choose a precise direction of plane in order to fix completely the notations. So, let $x^{\prime}$ belong to a plane in 
$\pi^{\perp}\left(\Sigma_{2}\right)$ parallel to $\left\{e_{1}^{\prime}, e_{5}^{\prime}\right\}$. Then the fiber $\pi^{\perp-1}\left(x^{\prime}\right)$ intersects in addition to the previous dodecahedra coming from $\Sigma_{1}$ a new set of rhombohedra coming from the internal partitions of the tiles $C_{I}$. We shall describe them through a strip projection: as already mentioned, we get a rhombohedron $\pi^{\prime \prime}\left(\gamma_{I}\right)+\xi$ for all $\xi$ such that $\pi^{\perp-1}\left(x^{\prime}\right)$ intersects all the boundaries (including the internal ones) of the rhombohedron $\pi^{\perp}\left(\gamma_{I^{c}}\right)$, and these $\xi$ are selected through a strip with a profile $x^{\prime}+\partial \pi^{\perp}\left(\gamma_{I^{c}}\right)$ (here the symbol $\partial$ refers to all the boundaries, including the internal ones.) Since we are interest only in the cells parallel to the direction $\left\{e_{1}^{\prime}, e_{5}^{\prime}\right\}$, let us enumerate the relevant rhombohedra. We know already the set of rhombohedra belonging to $\Sigma_{1}$, and we are interested now in the contribution of the internal partitions. They fall in two sets: those which are spanned by a edge and a diagonal of a facet, and those which are orthogonal to a facet of the rhombohedron. Let us deal separately with each type.

The two diagonals of facet parallel to the plane $\left\{e_{1}^{\prime}, e_{5}^{\prime}\right\}$ are $e_{3}^{\prime}-e_{6}^{\prime}$ and $e_{2}^{\prime}-e_{4}^{\prime}$, which are found in the four rhombohedra $\left\{e_{1}^{\prime}, e_{2}^{\prime}, e_{4}^{\prime}\right\},\left\{e_{5}^{\prime}, e_{2}^{\prime}, e_{4}^{\prime}\right\},\left\{e_{1}^{\prime}, e_{3}^{\prime}, e_{6}^{\prime}\right\},\left\{e_{5}^{\prime}, e_{3}^{\prime}\right.$, $\left.e_{6}^{\prime}\right\}$, and for each of them, we get in the fiber a rhombohedron spanned by the "complementary" vectors. It will be convenient to consider these last rhombohedra as attached to vertices falling under $\pi^{\perp}$ on the symmetry plane $\mathbb{Q}$ parallel to $\left\{e_{1}^{\prime}, e_{5}^{\prime}\right\}$ of the triacontahedron $x^{\prime}+\pi^{\perp}\left(\gamma_{6}\right)$. We obtain in this way (the notation $\{u, v\}$ denotes as usual the parallelogram spanned by the vectors $u$ and $v$ ):

- the rhombohedron $\left\{-e_{3}, e_{5}, e_{6}\right\}$ through the strip $x^{\prime}+e_{3}^{\prime}+e_{4}^{\prime}+\left\{e_{1}^{\prime}, e_{2}^{\prime}-e_{4}^{\prime}\right\}$; - the rhombohedron $\left\{e_{1},-e_{3}, e_{6}\right\}$ through the strip $x^{\prime}+e_{2}^{\prime}+e_{3}^{\prime}+\left\{e_{5}^{\prime}, e_{4}^{\prime}-e_{2}^{\prime}\right\}$; - the rhombohedron $\left\{-e_{2}, e_{4}, e_{5}\right\}$ through the strip $x^{\prime}+e_{2}^{\prime}+e_{3}^{\prime}+\left\{e_{1}^{\prime}, e_{6}^{\prime}-e_{3}^{\prime}\right\}$; - the rhombohedron $\left\{-e_{2}, e_{4}, e_{1}\right\}$ through the strip $x^{\prime}+e_{2}^{\prime}+e_{3}^{\prime}+\left\{e_{5}^{\prime}, e_{6}^{\prime}-e_{3}^{\prime}\right\}$. The second set of internal boundaries comes from the symmetry planes of the dodecahedra which are orthogonal to their long diagonal. We find such boundaries parallel to $\left\{e_{1}^{\prime}, e_{5}^{\prime}\right\}$ in the four rhombohedra $\left\{e_{3}^{\prime}, e_{4}^{\prime}, e_{6}^{\prime}\right\},\left\{e_{2}^{\prime}, e_{3}^{\prime}, e_{6}^{\prime}\right\},\left\{e_{2}^{\prime}\right.$, $\left.e_{4}^{\prime}, e_{6}^{\prime}\right\},\left\{e_{2}^{\prime}, e_{3}^{\prime}, e_{4}^{\prime}\right\}$. Thus we find in the fiber the corresponding rhombohedra which are the four rhombohedra with the facet $\left\{e_{1}, e_{5}\right\}$ in their boundary. The profiles of the associated strips are not so simple to set out: they are made for the thick rhombohedron of its intersection with two planes orthogonal to a facet direction going through the small diagonal of the two corresponding facets, and for the thin rhombohedron of its analogous intersections with two planes going through the long diagonal of two parallel facets. Observe that these intersections come by parallel pairs: for instance, one component in the rhombohedron $\left\{e_{3}^{\prime}, e_{4}^{\prime}, e_{6}^{\prime}\right\}$ is mapped by the translation $e_{2}^{\prime}$ on a component of the rhombohedron $\left\{e_{2}^{\prime}, e_{3}^{\prime}, e_{6}^{\prime}\right\}$. Since similar considerations hold for the other component of the same rhombohedron and for the other pair of rhombohedra, one can keep only one component for each rhombohedron, and consider it as the profile of a strip selecting pairs of rhombohedra, each pair being made of two rhombohedra symmetric with respect to the plane $\left\{e_{1}, e_{5}\right\}$ and sharing a facet falling in this plane.

Finally, one can synthesize the construction as follows: Consider the dodecahedron $x^{\prime}+\left\{e_{2}^{\prime}, e_{3}^{\prime}, e_{4}^{\prime}, e_{6}^{\prime}\right\}$, (the existence domain of the facet $\left\{e_{1}, e_{5}\right\}$ ) together with its tiling by the four previous rhombohedra. Then the profiles we are looking for are the intersections of these rhombohedra with the symmetry plane $\mathbb{Q}$, and since $\mathbb{Q}$ is also a symmetry plane for the dodecahedron, their union is easily seen to be a rectangle. We will content ourselves with this description. 


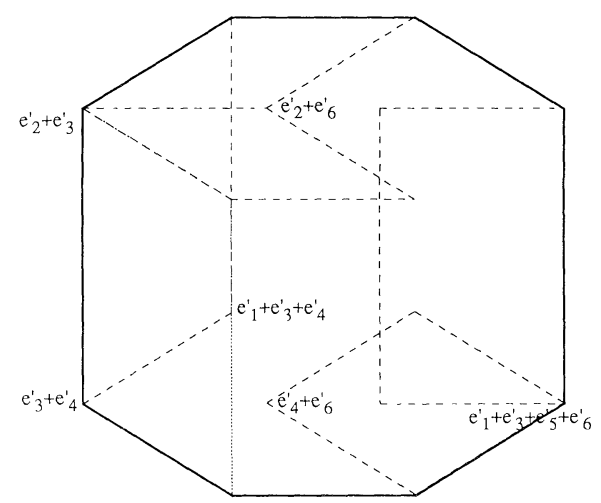

Fig. 8. The profile of the strip defining the quasiperiodic tilings of $\mathbb{P}$
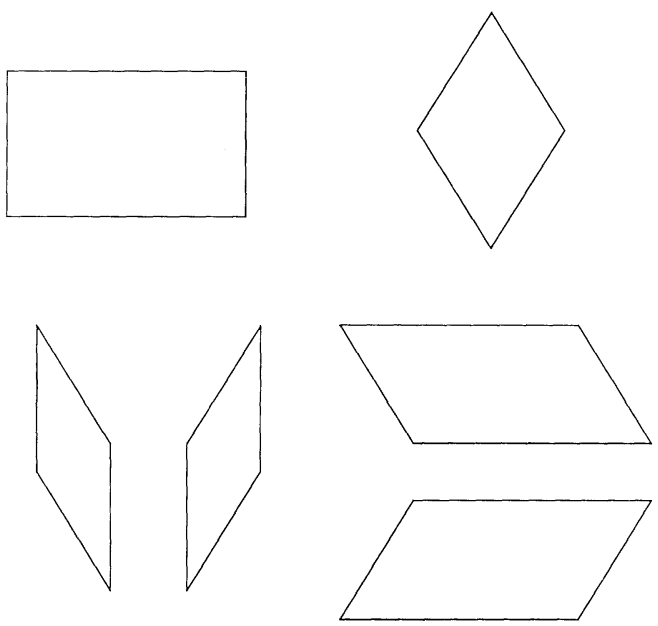

Fig. 9. The six tiles of the tiling of $\mathbb{P}$

The next step is to show that the union of all these rhombohedra and of the dodecahedra coming from $\Sigma_{1}$ covers a plane and thus can be retracted onto this plane. This plane is the affine support $\mathbb{P}$ of $\varphi\left(\mathbb{Q} \cap \pi^{\perp}\left(\mathbb{Z}^{6}\right)\right)$ along which are distributed all our polyhedra. To do this, we define a quasiperiodic tiling of this plane and we show that the tiles are the intersections of the plane with our polyhedra. Let us consider the 4-dimensional plane $\mathbb{I I I}$ in $\mathbb{R}^{6}$ spanned by $\left\{\varepsilon_{1}, \varepsilon_{5}\right.$, $\left.\varepsilon_{4}-\varepsilon_{2}, \varepsilon_{6}-\varepsilon_{3}\right\}$ which projects on $\mathbb{Q}$ in $\mathbf{E}^{\perp}$ and on $\mathbb{P}$ in $\mathbf{E}^{\|}$. We construct a tiling of $\mathbb{P}$ through a strip projection involving the traces of $\mathbb{Z}^{6}$ and $\gamma_{6}$ on IIII. One easily verifies that the profile of the corresponding strip is precisely the intersection of the triacontahedron with $\mathbb{Q}$ (Fig. 8), and that we get in this way a tiling by means of six tiles depicted on Fig. 9: they are a rhomb, a rectangle, and two pairs of symmetric parallelograms. Now, all we have to do is to observe that these tiles are the traces of our polyhedra on $\mathbb{P}$ : the rectangle is the intersection with $\mathbb{P}$ of the dodecahedra coming from $\Sigma_{1}$, the parallelograms are the traces of the first set of rhombohedra discussed above, and the rhombs, which are parallel to $\left\{e_{1}, e_{5}\right\}$, belong to the 
second set of rhombohedra, which all intersect $\mathbb{P}$ on their boundaries. Moreover, the existence domains of the tiles of this tiling of $\mathbb{P}$ exactly coincide with the profiles of the strips selecting the corresponding rhombohedra (see Fig. 8): the rhomb is on one hand the existence domain of the rectangle and on the other the profile of the strip which selects the dodecahedra of $\Sigma_{1}$, each parallelogram is the domain of a parallelogram of the same type and the profile of the strip which selects the relevant rhombohedra, and finally the rectangle is the domain of the rhombs and the union of the strips selecting the pairs of rhombohedra whose common facets falling on $\mathbb{P}$ are the rhombs. This proves that the plane $\mathbb{P}$ is completely covered by the intersection $\Sigma_{2} \cap \pi^{\perp-1}\left(x^{\prime}\right)$. Thus we see that this set can be retracted on the plane $\mathbb{P}$, which proves the lemma.

As a remark, observe that we can reorganize this set of rhombohedra much as we have done for $\Sigma_{1}$. We get in this way a quasiperiodic packing of the three orientations of dodecahedra for which $\mathbb{P}$ is a symmetry plane, which covers $\mathbb{P}$ without holes. Moreover, when $x^{\prime}$ moves on the plane $\mathbb{Q}-e_{2}^{\prime}-e_{3}^{\prime}$, we immediately see that for each $x^{\prime}$ we get another tiling of $\mathbb{P}$, in such a way that the tiling changes with $x^{\prime}$, but not the plane it covers. We thus get an "homotopy skeleton" of $\Sigma_{2}$ which is very simple: it is a union of 4-dimensional planes parallel to III, and to its images through the icosahedral group.

Let us describe the situation when the point $x^{\prime}$ lies on the intersection of two or more of the planes of $\Sigma_{2}$. In such a case, we have to consider several symmetry planes $\mathbb{Q}$ and their images $\mathbb{P}$. Since these planes are parallel to the fifteen symmetry planes of the triacontahedron, it is clear that they intersect either on a two-fold axis of the triacontahedron or on its center, and this is the second key of our theorem.

Observe that when these symmetry planes intersect on a point, then this point is either in $\pi^{\perp}\left(\mathbb{Z}^{6}\right)$ or in the projection of the centers of the hypercubes of $\mathbb{R}^{6}$, and we find at these points the whole star of fifteen planes. In each fiber of $\pi^{\perp}$ above a point belonging to an axis, we find the corresponding union of packings of tiles covering their respective planes, plus other tiles whose vertices come from the edges of the triacontahedron $x^{\prime}+\gamma_{6}$ or from the intersections of the internal partitions, and which "thicken" the intersection of the planes. We do not need to describe these extra tiles since they do not change anything to the homotopy type of $\Sigma_{2} \cap \pi^{\perp-1}\left(x^{\prime}\right)$, which is represented by the union of 2-dimensional planes. Finally, one verifies that above the projections of vertices or of centers of hypercubes of $\mathbb{Z}^{6}$, the set $\Sigma_{2}$ $\cap \pi^{\perp-1}\left(x^{\prime}\right)$ has the homotopy type of the union of the fifteen symmetry planes intersecting on a point.

7.2. We can now state our main theorem:

Theorem. Any tiling of the three dimensional space by Ammann rhombohedra with matching decorations is a quasiperiodic Penrose tiling.

Proof. We shall compute the homology group $H_{3}\left(\mathbb{R}^{6}-\left(\Sigma_{2} \cap \mathbb{U}\right)\right)$. As shown in Sect. 5, it is isomorphic to the group $H_{2}\left(\Sigma_{2} \cap \mathbb{U}\right)$, and using the previous lemma, this group is very easy to compute.

Let us define a retraction by deformation of $\Sigma_{2} \cap \mathbb{U}$ onto a 2-dimensional cellular complex. As is well known, such a retraction is an homotopy equivalence and preserves the homology. We define our retraction by "gluing" together a family of homotheties which retract each fiber of $\pi^{\perp}$ on a point, and for this we 
have to choose an homothety center in each fiber. Observe that in the fibers with a star of fifteen planes we have no choice, since the unique possible homothety center is the point on which the planes intersect. Now, observe that since along any symmetry axis starting from such a point in $\mathbf{E}^{\perp}$, we find another point of the same kind, it is natural to define the homothety center on each fiber above the segments thus defined on any symmetry axis, through a linear interpolation between the endpoints of these segments, which yields a point on the intersection of planes in the fiber, and finally since these symmetry axes define a triangulation of each plane in $\pi^{\perp}\left(\Sigma_{2} \cap \mathbb{U}\right)$, we choose the center on the generic fibers through a linear interpolation between the vertices of the triangles, which yields of course a point in our plane in the fiber.

It is clear that this construction provides us with a continuous retraction of $\Sigma_{2} \cap \mathbb{U}$ onto a 2-dimensional complex. Now, observe that the restriction of $\pi^{\perp}$ to this complex is one to one, since we have exactly one point in each fiber. Moreover, our choice of the homothety centers entails the continuity of the inverse map $\pi^{\perp-1}$. This means that this complex is isomorphic to $\pi^{\perp}\left(\Sigma_{2} \cap \mathbb{U}\right)$, which is what we need.

In fact, it is obvious that $H_{2}\left(\pi^{\perp}\left(\Sigma_{2} \cap \mathbb{U}\right)\right.$ is generated by the boundary of each cell it defines in $\mathbf{E}^{\perp}$, so we obtain through duality by intersection a basis of $H_{3}\left(\mathbb{R}^{6}-\left(\Sigma_{2} \cap \mathbb{U}\right)\right)$ made of classes which cross each cell only once. This classes can be realized by a piece of a plane parallel to $\mathbf{E}^{\|}$going through $\mathbb{U}$, closed outside $\mathbb{U}$ by, say, a 3-dimensional hemisphere. This basis is very well suited for our purposes since it is composed of classes whose intersection indices with the triacontahedra $\sigma_{\xi}$ for $\xi \in \mathbb{Z}^{6} \cap \mathbb{U}$ is 0 or 1 : we conclude that we have classified with this basis all the packing of tiles with matching decorations coming from hypercubes contained in $\mathbb{U}$.

To complete the proof, we have now only to observe that any infinite cut must yield such a restriction to each $\mathbb{U}$. But since the cells of $\pi^{\perp}\left(\Sigma_{2} \cap \mathbb{U}\right)$ shrink to zero when $\mathbb{U}$ becomes larger and larger, this is possible if and only if the cut is a union of planes, and each of these planes defines a quasiperiodic Penrose tiling. Q.E.D.

\section{Comments}

As one can see, the structure of the proof is in fact simple, and our approach is clearly not restricted to the 3-dimensional Penrose tilings. There are three steps in the general case (which involves a projection from $\mathbb{R}^{n}$ onto $\mathbb{R}^{p}$ for $p$-dimensional tilings): the first one is to express the matching rules through a "forbidden set" which must not be intersected by the cut selecting the vertices, and this is the hard core of our method. The second step is to compute the $p$-th homology group of the complementary in $\mathbb{R}^{n}$ of this forbidden set, in order to classify all the possible packings of tiles defined by such cuts. Finally, to prove that the matching rules force the quasiperiodicity of the tilings, it remains to show that in each homology class which defines a tiling, there is a p-dimensional plane: since these packings of tiles are not whole tilings in general, we have to sort out the homology classes which correspond to tilings (which are packings without holes nor overlappings). This is a difficult task in the general case, and we do not know any characterization of these classes: for instance, a cut will surely define a tiling if it is a section (avoiding the forbidden set) of the fiber bundle

$$
\mathbb{R}^{n} \stackrel{\pi \|}{\longrightarrow} \mathbf{E}^{\|}
$$

but this sufficient condition is not necessary. 
It is important to observe that for the matching rules discussed here, this last step is in fact trivial: we have not to worry about those classes which correspond to tilings, since the computation of the homology group directly exhibits a basis made of classes which contain a plane. It is then evident that the classes which define a tiling are simply the elements of this basis, with a coefficient +1 . Technically, this follows from the strong property that the projection $\pi^{\perp}\left(\Sigma_{2}\right)$ of the forbidden set is a deformation retract of $\Sigma_{2}$ [recall that this retraction is the composition of a first retraction of $\Sigma_{2}$ onto a set of 4-dimensional planes, followed by a second retraction on one point in each fiber of $\pi^{\perp}$ and the projection onto $\left.\pi^{\perp}\left(\Sigma_{2}\right)\right]$.

This situation results in a major simplification and it may be useful to describe the difficulties which arise when this third step is no longer trivial. We shall briefly examine the case of a simpler decoration of the 3-dimensional Penrose tilings and the case of the (generalized) 2-dimensional Penrose tilings.

A simpler set of decorations of the two Ammann rhombohedra is readily obtained from ours by forgetting about the colour of the marks. This corresponds to a decomposition of the rhombic dodecahedron in only four sectors, involving the two symmetry planes which contain the main diagonal of the dodecahedron, and results in only two different decorated rhombohedra of each type.

The study of the forbidden set $\Sigma_{2}$ corresponding to these new matching rules runs as in the previous case, and yields of course a smaller set: specifically, the set $\Sigma_{2} \cap \pi^{\perp-1}\left(x^{\prime}\right)$ (above an $x^{\prime}$ belong to only one symmetry plane) does not cover completely the plane $\mathbb{P}$, and it is immediate that the "holes" are the rhombs of Fig. 9, whose existence domain is the rectangle in Fig. 8, since these contributions to $\Sigma_{2}$ precisely come from the internal partitions of the rhombic dodecahedra which are orthogonal to the main diagoal and which are missing in these simpler matching rules.

Accordingly, we find holes in the 4-dimensional planes on which is retracted $\Sigma_{2}$, and the main question concerns the topology of these holes: of course, the projection $\pi^{\perp}\left(\Sigma_{2}\right)$ will no longer be homotopic to $\Sigma_{2}$, but we need only the equality of their second homology group to conclude, so that we have to examine if we change this $\mathrm{H}_{2}$ when we "fill" these holes.

Using for instance the Mayer-Vietoris sequence, it is easy to see that we do not change the $\mathrm{H}_{2}$ if the holes which we fill in the 4-dimensional planes are topologically 4-dimensional balls, but we do change the $H_{2}$ if these holes have the homotopy type of a cylinder $B_{3} \times \mathbb{R}$, where $B_{3}$ is a 3 -dimensional ball, since its boundary gives a contribution to $\mathrm{H}_{2}\left(\Sigma_{2}\right)$ which vanishes upon the filling. Now, one can verify by a careful inspection of the local patterns in the tiling of $\mathbb{P}$ that this is precisely what happens: the holes glue together to form a set of cylinders $B_{3} \times \mathbb{R}$, and are thus large enough for a cut to "escape" through them.

From a purely geometrical point of view, one can describe the situation as follows: a 3-dimensional cut and a 4-dimensional plane generically intersect in $\mathbb{R}^{6}$ along a one dimensional line, so that an hole in the 4-dimensional plane large enough for the cut to get through must extend at least along a whole line, and this is what happens in our case.

These considerations suggest that the simpler matching rules do not force the quasiperodicity. However, let us stress that they do not constitute a proof of anything, for at least two reasons: we have not studied what happens on the intersections of the 4-dimensional planes, and we have not shown that the cuts 
which "escape" through the holes actually define tilings without overlappings nor holes. To get a definitive answer on this question requires more work (see also [13] and [14] for tentative simple matching rules).

Our last comment will concern the 2-dimensional Penrose tilings. As is well known (see for instance [9]), they can be obtained through a projection technique which we recall very briefly below: Start with $\mathbb{R}^{5}$ equipped with the hypercubic lattice $\mathbb{Z}^{5}$ and consider the group which permutes the five basis vectors. When decomposing this group action into real irreducible representations, one finds one invariant line (the main diagonal of $\mathbb{R}^{5}$ ) and two invariant planes. Then choose one of these planes as $\mathbf{E}^{\|}$and the sum of the other plane and of the diagonal as $\mathbf{E}^{\perp}$. The strip construction along $\mathbf{E}^{\|}$yields tilings of $\mathbf{E}^{\|}$by means of the projections of the ten 2-dimensional facets of the hypercubic lattice, which are the two Penrose rhombs, each of which being obtained in five different orientations. The profile $\mathbf{K}$ of the strip is as usual the projection on $\mathbf{E}^{\perp}$ of the unit hypercube. It is easily seen to be a rhombic icosahedron, the parallelohedron spanned by the five vectors $e_{i}^{\prime}$, $i=1, \ldots, 5$ of Fig. 2 .

There is a specific feature in this construction, which does not exist in the icosahedral case, and which comes from the fact that the projection $\pi^{\perp}\left(\mathbb{Z}^{5}\right)$ is not homogeneous in $\mathbf{E}^{\perp}$ : since $\mathbf{E}^{\perp}$ intersects $\mathbb{Z}^{5}$ along a 1 -dimensional lattice (carried by the diagonal), $\pi^{\perp}\left(\mathbb{Z}^{5}\right)$ falls on a set of equidistant planes orthogonal to the five fold axis of the icosahedron (which is parallel to the diagonal). As a consequence, the properties of the tilings depend on the position of the icosahedron $\mathbf{K}_{\alpha}$ with respect to this set of planes, and they fall in classes known as "local isomorphism classes" (see [14]), which are classified by the position of $\mathbf{K}_{\alpha}$ along the diagonal. In particular, the original Penrose tilings, for which matching rules are known and which present strong self similarity properties, are obtained for the translations $\alpha$ such that the vertices of the icosahedron $\mathbf{K}_{\alpha}$ fall on the previous set of planes. The other positions correspond to the so-called generalized Penrose tilings.

Let us now turn to the decoration of the rhombs, whose construction is completely analogous to the icosahedral case: we have to decorate the edges of the rhombs, and the existence domains of these edges in the icosahedron are again rhombic dodecahedra. One can easily verify that in order to recover the de Bruijn's arrowing of the Penrose rhombs, one has to partition the dodecahedra in four sectors defined by the two symmetry planes intersecting on the long diagonal of the dodecahedron: on account of the symmetry requirements, one finds that there are to kinds of marks, which can be identified with the single and double arrows of de Bruijn.

The next step is to obtain the decorated rhombs which result from these decorations of the edges. The existence domains of the rhombs are rhombohedra, and one can see that each rhombohedron is partitionned into four subcells by the partitions of the dodecahedra attached to the edges of the rhomb. On account of the symmetry, one gets only two decorated rhombs of each type: one pair are the classical decorated Penrose rhombs, and the other pair was first introduced in [15].

Finally, one can observe that the geometry of the existence subdomains of decorated tiles in the icosahedron is such that when the icosahedron has its vertices on the planes which carry $\pi^{\perp}\left(\mathbb{Z}^{5}\right)$, then only the subdomains of the two decorated 
Penrose rhombs are intersected by these planes. Thus we recover the well known fact that the original Penrose tilings can be decorated using only one thick and one thin decorated rhomb, while one needs two thick and two thin decorated rhombs to decorate the generalized Penrose tilings with matching decorations (see [15]).

However, our main concern is with the converse question: is this decoration of the rhombs sufficient to force the quasiperiodicity of the tilings? As for the icosahedral case, we switch to the corresponding cut description and we study the set $\Sigma_{2}$ associated to these matching rules, through its traces on the fibers of $\pi^{\perp}$, which are planes parallel to $\mathbf{E}^{\|}$.

We give only the results which we have obtained, and we encourage the reader to work out the details: this is essentially a paraphrase of the previous discussion of the icosahedral case. Since for a generic $x^{\prime}$ in $\mathbf{E}^{\perp}$, the intersection $\Sigma_{2} \cap \pi^{\perp-1}\left(x^{\prime}\right)$ is empty, we have first to find the projection $\pi^{\perp}\left(\Sigma_{2}\right)$ : it is made of a set of ten dense families of planes, parallel to the ten directions of facets of the icosahedron. For an $x^{\prime}$ belonging to only one of these planes, we look for the set $\Sigma_{2} \cap \pi^{\perp-1}\left(x^{\prime}\right)$. This intersection is a collection of rhombs obtained with a relevant strip, and they are arranged in a "ribbon" which covers a whole infinite line in the fiber, much like the corresponding set of rhombohedra covers a whole plane in the icosahedral case. Let us remark here that the matching rules appear sufficiently strong, in the sense that if we add more constraints to them (for instance by taking into account the third symmetry plane of the dodecahedron like in the 3-dimensional case) we do not change the homotopy type of $\Sigma_{2} \cap \pi^{\perp-1}\left(x^{\prime}\right)$, but we only thicken the patch of tiles which will still cover the same infinite line. Thus we do not find the kind of difficulty that we have encountered with the simplified matching rules in the 3-dimensional case.

The next step is to examine the situation when the point $x^{\prime}$ is on the intersection fo two planes. Of course, we find in the fiber $\pi^{\perp-1}\left(x^{\prime}\right)$ the union of the two "ribbons" corresponding to each plane, and the relative position of the two ribbons depends on the pair of planes under consideration. More precisely, one can observe that there are five pairs of planes such that these ribbons are parallel: they are the pair $\left(\left\{e_{1}^{\prime}, e_{2}^{\prime}\right\},\left\{e_{3}^{\prime}, e_{5}^{\prime}\right\}\right)$ and the four other pairs obtained through the five-fold symmetry. Let us now explain why this situation breaks down our machinery.

Recall that the key of our approach is that the forbidden set $\Sigma_{2}$ has the same homotopy type that its projection on $\mathbf{E}^{\perp}$. In the icosahedral case, this follows from the fact that we can for any $x^{\prime}$ retract $\Sigma_{2} \cap \pi^{\perp-1}\left(x^{\prime}\right)$ onto a point, and this is possible because for any $x^{\prime}$ this set is (homotopically) a union of concurrent planes, and thus is a contractible set. This is no longer the case for the 2-dimensional Penrose tilings: in some fibers we find a pair of parallel lines, in others we find a set of nonconcurrent lines, which can be retracted on a polygon but not on a point. Then it is not true that the group $H_{2}\left(\Sigma_{2}\right)$ is isomorphic to $H_{2}\left(\pi^{\perp}\left(\Sigma_{2}\right)\right)$ and we cannot conclude. On the other hand, we cannot claim that these decorations do not force the quasiperiodicity of the tilings (although this seems likely) because we do not know how to characterize the classes in $H_{2}\left(\Sigma_{2}\right) \approx H_{2}\left(\mathbb{R}^{5}-\Sigma_{2}\right)$ which correspond to tilings.

As a last remark on this question, let us mention that the special behaviour of the original Penrose tilings is linked with the following: when the point $x^{\prime}$ belongs to one of the planes on which falls $\pi^{\perp}\left(\mathbb{Z}^{5}\right)$, and simultaneously to two planes of 
$\pi^{\perp}\left(\Sigma_{2}\right)$ giving parallel ribbons, then these two ribbons overlap in such a way that their union has the homotopy type of a single line and that we can again retract $\Sigma_{2}$ $\cap \pi^{\perp-1}\left(x^{\prime}\right)$ onto a point. With this remark and the constraint of using only the two decorated Penrose rhombs to build a tiling, it seems possible to prove the quasiperiodicity of the original Penrose tilings within our framework. Since this property is already known as a consequence of the work of de Bruijn (see [2] and [9]), we have not worked out the details.

Acknowledgements. This work was supported in part by the National Science Foundation under Grant $n^{\circ}$ PHY 82-17853, supplemented by funds from the National Aeronautics and Space Administration.

\section{References}

1. Penrose, R.: Math. Intel. 2, 32-37 (1979)

2. de Bruijn, N.G.: Nederl. Akad. Wtensh. Proc. Ser. A 43, 39-66 (1981)

3. Garnder, M.: Sci. Am. 236, No. 1, 110 (1977)

4. Grünbaum, B., Shephard, G.C.: Tilings and patterns. Oxford: W. H. Freeman 1987

5. Mackay, A.L.: Physica 114 A, 66 (1982)

6. Shechtman, D., Blech, I., Gratias, D., Cahn, J.W.: Phys. Rev. Lett. 53, 1951 (1984)

7. Les Houches, Workshop on Aperiodic Crystals. J. Phys. France 47, C3 (1986)

8. Kramer, P., Neri, R.: Acta Crystallogr. Sec. A 40, 580 (1984)

9. Katz, A., Duneau, M.: J. Phys. France 47, 181-196 (1986)

10. Oguey, C., Duneau, M., Katz, A.: A geometrical approach of quasiperiodic tilings. Commun. Math. Phys. 118, 99-118 (1988)

11. Bak, P.: Phys. Rev. Lett. 56, 861 (1986)

12. Dress, A.: Private communication

13. Socolar, J., Steinhardt, P.J.: Phys. Rev. B 34, 617 (1986)

14. Levine, D.: J. Phys. France 47, C3, 125 (1986)

15. Kleman, M., Pavlovitch, A.: J. Phys. France 47, C3, 229 (1986)

Communicated by A. Jaffe

Received November 10, 1987; in revised form March 18, 1988 